\title{
The Ogievetsky-Polubarinov massive gravity and the benign Boulware-Deser mode
}

\section{Shinji Mukohyama ${ }^{a, b}$ Mikhail S. Volkov ${ }^{c, d}$}

\author{
${ }^{a}$ Center for Gravitational Physics, Yukawa Institute for Theoretical Physics, Kyoto \\ University, 606-8502, Kyoto, Japan \\ ${ }^{b}$ Kavli Institute for the Physics and Mathematics of the Universe (WPI), The Uni- \\ versity of Tokyo Institutes for Advanced Study, The University of Tokyo, Kashiwa, \\ Chiba 277-8583, Japan \\ ${ }^{c}$ Institut Denis Poisson, UMR - CNRS 7013, \\ Université de Tours, Parc de Grandmont, 37200 Tours, France \\ ${ }^{d}$ Department of General Relativity and Gravitation, Institute of Physics, \\ Kazan Federal University, Kremlevskaya street 18, 420008 Kazan, Russia \\ E-mail: shinji.mukohyama@yukawa.kyoto-u.ac.jp, \\ michael.volkov@idpoisson.fr
}

\begin{abstract}
.
We present our analysis of the theory constructed in 1965 by Ogievetsky and Polubarinov (OP) - the first ever theory of interacting massive gravitons. Its mass term is adjusted in such a way that the non-linear field equations imply as a consequence the linear Hilbert-Lorentz condition, which restricts the spin of states in the theory. Strikingly, for special parameter values this theory coincides with one of the "ghost-free" massive gravity models rediscovered only in 2010. For generic parameter values, however, it propagates 6 degrees of freedom and shows ghost around flat space. Surprizingly, we find that the de Sitter space remains stable for a large region of the parameter space, provided that the Hubble expansion rate is large enough, hence the Boulware-Deser mode is benign in this case. We study also other solutions and find that the Milne universe - a sector of Minkowski space - is stable in the UV limit. This presumably implies that at the non-linear level the ghost instability in flat space develops only for long waves, similarly to the classical Jeans instability.
\end{abstract}




\section{Contents}

1 Introduction 1

2 The OP theory $\quad 5$

$\begin{array}{lll}2.1 & \text { Field equations } & 7\end{array}$

2.2 Subsidiary conditions 8

3 Solutions with proportional metrics $\quad 10$

4 Stability conditions $\quad 13$

4.1 The FP limit 15

$\begin{array}{lll}4.2 & \text { Generic massive gravity } & 16\end{array}$

5 More general cosmologies $\quad 16$

$\begin{array}{lll}5.1 & \text { Stability conditions } & 17\end{array}$

$\begin{array}{lll}5.2 & \text { Simplest solutions } & 18\end{array}$

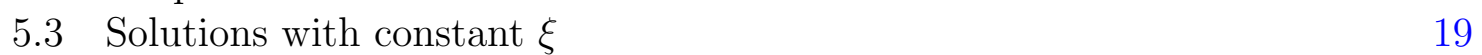

5.4 Solutions with Minkowski fiducial metric 20

5.5 Solutions with Milne fiducial metric 24

6 Summary and concluding remarks $\quad 25$

A Stability of more general cosmologies $\quad 26$

\section{Introduction}

In this paper we analyze the massive gravity theory constructed in 1965 by Ogievetsky and Polubarinov (OP) [1] $]^{1}$. To our knowledge, this had been the first serious work on massive gravity after Fierz and Pauli [2], and the first ever systematic study of interacting massive gravitons. Among other things, OP obtained one of the "ghostfree" massive gravity models rediscovered again only in 2010 [3]. However, their work is almost unknown in the modern massive gravity community, presumably because their strategy was quite different from what is generally adopted at present. Therefore, in what follows we shall present our analysis of the OP theory and of some of its applications.

To understand the OP's motivations, consider free massive gravitons in Minkowski space described by a symmetric tensor $\Psi_{\mu \nu}$ subject to [2]

$$
\begin{aligned}
\left(\partial^{\sigma} \partial_{\sigma}-m^{2}\right) \Psi_{\mu \nu} & =0 \\
\partial^{\sigma} \Psi_{\sigma \mu} & =0 \\
\Psi_{\sigma}^{\sigma} & =0 .
\end{aligned}
$$

\footnotetext{
${ }^{1}$ V. I. Ogievetsky and I. V. Polubarinov worked in Dubna in the Soviet Union times.
} 
The tensor $\Psi_{\mu \nu}$ has 10 independent components, but the five conditions in (1.2) and (1.3) eliminate the spin- 1 and two spin-0 representations and there remains only the massive spin-2 carrying $5=10-5$ degrees of freedom (DoF). As emphasized already by Fierz and Pauli (FP) [2], the Lorentz condition (1.2) is absolutely essential, because if it were replaced by some other four conditions to keep the same number of DoF, the canonical energy of $\Psi_{\mu \nu}$ would be non-positive.

The OP's goal was to find a non-linear completion for Eqs.(1.1)-(1.3). They adopted the field-theoretical approach initiated by Papapetrou [4], Gupta [5], and Feynman [6], and considered gravitons as interacting fields in flat space. Therefore, they kept $\Psi_{\mu \nu}$ (or rather $\Psi^{\mu \nu}$ ) as the principle variables and were looking for non-linear terms to be added to (1.1) to describe the graviton interactions.

To illustrate the idea, let us consider the massless case - the General Relativity. It is well known that the Einstein equations can be represented in the Papapetrou form (see, e.g. [7]) as

$$
\partial_{\mu} \partial_{\nu}\left(\mathfrak{g}^{\alpha \beta} \mathfrak{g}^{\mu \nu}-\mathfrak{g}^{\alpha \nu} \mathfrak{g}^{\beta \mu}\right)=16 \pi G(-g) t_{\mathrm{LL}}^{\alpha \beta},
$$

where $\mathfrak{g}^{\mu \nu}=\sqrt{-g} g^{\mu \nu}$ and the Landau-Lifshitz pseudo-tensor $t_{\mathrm{LL}}^{\alpha \beta}$ does not contain second derivatives. It is always possible to impose the harmonic gauge condition $\partial_{\mu} \mathfrak{g}^{\mu \nu}=0$. Introducing the tensor $\Psi^{\mu \nu}$ via

$$
\sqrt{-g} g^{\mu \nu}=\eta^{\mu \nu}+\Psi^{\mu \nu}
$$

Eqs.(1.4) assume the form

$$
\begin{aligned}
\partial^{\sigma} \partial_{\sigma} \Psi^{\mu \nu} & =\left(\text { terms non-linear in } \Psi^{\mu \nu}\right), \\
\partial_{\sigma} \Psi^{\sigma \nu} & =0 .
\end{aligned}
$$

These equations can be viewed as describing gravitons in Minkowski space. The nonlinear terms on the right in (1.6) describe graviton interactions. In the linear approximation one neglects the interaction terms and the equations describe free gravitons,

$$
\begin{aligned}
\partial^{\sigma} \partial_{\sigma} \Psi^{\mu \nu} & =0 \\
\partial_{\sigma} \Psi^{\sigma \nu} & =0 .
\end{aligned}
$$

One can then wonder if it is possible to go back from these linear equations to the nonlinear ones (1.6),(1.7) and apply the field theory methods to reconstruct the interaction terms ? In other words, can one obtain the General Relativity as the non-linear completion for the theory of free gravitons, without relying on methods of differential geometry ? Today we know that this is indeed possible [8], but in 1965 this fact was not known.

Therefore, the OP's aim was to apply the field theory methods to construct nonlinear terms to be added to the right hand side of (1.1) to obtain a consistent selfinteracting theory. Remarkably, they achieved the goal and, starting from the very first principles, constructed a fully interacting theory whose action contains the EinsteinHilbert kinetic term and has also a graviton mass term. Sending the graviton mass to zero they recovered the General Relativity. Therefore, OP have been the first to 
obtain the General Relativity by applying only field-theory methods, without using the differential geometry ${ }^{2}$.

The central role in their construction is played by the subsidiary conditions (1.2) and (1.3). However, OP had realized that it would have been technically too difficult to keep both of them. Therefore they imposed only one combined Hilbert-Lorentz condition,

$$
\partial_{\mu}\left(\Psi^{\mu \nu}+q \eta^{\mu \nu} \Psi_{\alpha}^{\alpha}\right)=0
$$

with constant $q$. This is necessary, although not sufficient, for exclusion of negative energies. They called this condition "spin limitation principle". It excludes the spin-1 and a spin-0, but not the second spin-0, hence there remain altogether 6 DoF. OP required the formula (1.10) to be exactly the same also in the presence of interactions: it should always contain partial and not covariant derivatives ${ }^{3}$. Therefore, the spin limitation condition always remains "clean" and removes precisely the spin- 1 and spin-0 in the strict representation theory sense. This is probably the most important moment: OP keep control over the spin content of their theory.

At this point, it is worth posing to compare the OP's strategy with the logic commonly adopted at present, according to which the "healthy" massive gravity theory has to have 5 DoF to avoid the Boulware-Deser (BD) ghost [9], hence it should contain 5 constraints [3]. For a flat background ${ }^{4}$ these constraints have the structure similar to that in Eqs.(1.2),(1.3) and they eliminate precisely the spin-1 and two spin-0 states. However, for arbitrary backgrounds the fifth constraint has a rather complex structure and it is not obvious what spin states it eliminates ${ }^{5}$. This suggests that for generic backgrounds the theory may propagate superpositions of states of different spins, even though the total number of DoF is always 5 . Therefore, the theory controls the number of DoF but does not seem to always control their spin contents, which might explain why it shows pathologies for some backgrounds [11-13].

The OP's strategy was quite different. They constructed a theory with 6 DoF and did not care about the BD mode (the ghost problem was not known at the time). Instead, they preferred to have control over the spin contents of their theory - it contains only the spin- 2 and a spin- 0 , while spin- 1 states are definitely excluded. Whether or not this makes sense is to be understood.

Getting back to their construction, OP required the condition (1.10) to be a differential consequence of the second order field equations. This requirement lead to certain identity relations for the Lagrangian, implying the existence of a local internal symmetry. By analyzing the structure of the symmetry generators, OP concluded that the symmetry must formally coincide with the spacetime diffeomorphism symmetry, viewed in their approach as the internal symmetry acting on gravitons in flat space. OP were then able to construct the interaction terms order by order by requiring

\footnotetext{
${ }^{2}$ The well-known paper [8] of Deser on a similar subject (considering only the massless case) appeared a few years after the OP's work. Deser used the bootstrap method, quite different from the OP's approach.

${ }^{3}$ This is indeed possible in a bimetric theory.

${ }^{4}$ More generally, for Einstein space backgrounds.

${ }^{5}$ Already the linearized version of the 5-th constraint is very complex; see Appendix B in [10].
} 
that the symmetry algebra closes. They ended up with a theory whose kinetic term coincides with the standard Einstein-Hilbert term for the "effective" metric $g_{\mu \nu}$ whose inverse $g^{\mu \nu}$ is related to the graviton field $\Psi^{\mu \nu}$ via the relation similar to (1.5),

$$
\left(\frac{\sqrt{-g}}{\sqrt{-\eta}}\right)^{s+1}\left(\left(\hat{g}^{-1}\right)^{n}\right)^{\mu \nu}=\eta^{\mu \nu}+\Psi^{\mu \nu}
$$

Here the parameters $s, n$ are real and the precise meaning of the matrix power will be specified below. Therefore, the spacetime metric $g_{\mu \nu}$ arises in their approach as a secondary object related to the primary graviton field $\Psi^{\mu \nu}$ in a very non-linear way, via (1.11). Notice that this transformation is invertible and can be resolved with respect to $g_{\mu \nu}$. The OP action contains also a mass term constructed from $g^{\mu \nu}$ and $\eta_{\mu \nu}$.

Once the OP theory is obtained, it can be formulated entirely in terms of $g_{\mu \nu}$ and $\eta_{\mu \nu}$, and then it can be viewed simply as a bimetric theory. It implies certain on-shell conservation conditions which, when expressed in terms of the variables $\Psi^{\mu \nu}$ defined by Eq.(1.11), assume the form of the linear "spin limitation principle". All of this will be explained below.

Summarizing, there are two aspects of the OP's work. First, it presents the first systematic derivation of the Einstein-Hilbert kinetic term starting from the free theory and applying only the field theory principles. This is, of course, a remarkable achievement for which OP should be fully credited, in our opinion. Secondly, their procedure gives also a particular mass term, but the status of this is less clear, since it gives rise to 6 DoF - a property considered today as unacceptable.

At the same time, the OP mass term is a part of the very carefully designed derivation procedure. For a one-dimensional subset of the parameter space it shows the FP property and propagates only 5 DoF around flat space. For one particular point of this FP subset the theory propagates 5 DoF even at the non-linear level and coincides with one of the ghost-free $\mathrm{dRGT}^{6}$ models. Therefore, the OP procedure gave in 1965 the result that was rediscovered again only in 2010 !

All of this suggests that the OP massive gravity deserves studying, even though it propagates in general 6 DoF. Therefore, we present in what follows our analysis of this theory and of its solutions. Skipping its derivation indicated above and described in the OP's paper [1], we come directly to the theory itself. In modern terms, this is a bimetric theory ${ }^{7}$ containing the dynamical metric $g_{\mu \nu}$ and a non-dynamical reference metric $f_{\mu \nu}$, with a specially designed interaction potential constructed from these metrics.

In Section II, we rewrite the theory in modern notation and explain how the linear "spin limitation condition" follows from the non-linear field equations. In brief, this is simply the condition for the tensor obtained by varying the action with respect to $f_{\mu \nu}$. This tensor is conserved on-shell, which is true in any bimetric theory, but only in the OP theory the conservation condition can be made linear by the non-linear field redefinition (1.11).

\footnotetext{
${ }^{6} \mathrm{dRGT}$ - after the names of authors of [3].

${ }^{7} \mathrm{~A}$ bimetric theory is any theory with two metrics. It can be a massive gravity if only one of the metrics is dynamical, or a bigravity if both metrics are dynamical.
} 
We then study in Section III the simplest solutions, such as the de Sitter or Minkowski, and explicitly show that there are 6 propagating DoF, unless for a onedimensional subset of the parameter space for which there are only 5 DoF. In Section IV we obtain the effective action for fluctuations and, surprisingly, find that the de Sitter space is completely free of ghosts and gradient instabilities for a large region of the parameter space, although the flat space always shows ghost away from the FP limit. This is, perhaps, our most interesting finding - the fact that the 6 -th polarization can be totally harmless.

We then proceed to study in Section V other homogeneous and isotropic cosmologies in the theory. We find many different types of such solutions, but unfortunately most of them are unstable. At the same time, it turns out that the Milne space - a sector of Minkowski space - is stable in the UV limit. This suggests that the ghost instability of the flat space develops only for long waves, similarly to the classical Jeans instability. Our conclusions are formulated in Section VI, while the Appendix contains the derivation of the stability conditions for the homogeneous and isotropic cosmologies.

Few words about the impact of the OP's work. In the older days it was mentioned in the massive gravity context [14-16]. The important special case in which the theory propagates only 5 DoF and coincides with the dRGT theory was studied by Maheshwari in 1972 [17] (see [18] for an interesting historical account). Nowadays it is cited by experts in various field theory domains (see for example [19-24]), but it is almost totally unknown to the modern massive gravity community. This has given us the motivation for writing this text.

\section{The OP theory}

The OP theory is a particular case of bimetric massive gravity. Any such theory is described by the dynamical metric $g_{\mu \nu}$ and a non-dynamical reference metric $f_{\mu \nu}$. There is no general rule for choosing the latter, for example one can set it to be the Minkowski metric, $f_{\mu \nu}=\eta_{\mu \nu}$, but one can just as well leave it unspecified for the time being.

The action of the theory is

$$
S=M_{\mathrm{Pl}}^{2} \int\left(\frac{1}{2} R(g)-m^{2} U+\mathcal{L}_{\text {matter }}\right) \sqrt{-g} d^{4} x,
$$

where $m$ is a mass parameter and $U$ is a scalar function constructed from products of $f_{\mu \nu}$ with the inverse of the physical metric, $g^{\mu \nu}$. Introducing the matrix $\hat{S}$ with components

$$
(\hat{S})^{\mu}{ }_{\nu} \equiv S_{\nu}^{\mu}=g^{\mu \sigma} f_{\sigma \nu}
$$

and using brackets to denote trace, $[\hat{S}]=S_{\sigma}^{\sigma}$, the potential can be any function of traces of powers of $\hat{S}$,

$$
U=U\left([\hat{S}],\left[\hat{S}^{2}\right],\left[\hat{S}^{3}\right], \operatorname{det}(\hat{S})\right)
$$


For example, it can be given by the series

$$
U=a_{0}+a_{1}[\hat{S}]+a_{2}\left[\hat{S}^{2}\right]+a_{3}[\hat{S}]^{2}+\ldots,
$$

where $a_{k}$ are constant coefficients. This theory generically shows 6 DoF in the gravity sector. If $a_{2}+a_{3}=0$ then the theory is said to fulfill the FP property and shows only 5 DoF around flat space. However, even then an extra 6-th polarization emerges when one deviates from flat space, unless the potential is further fine-tuned. This extra mode carries a negative kinetic energy and is called BD ghost [9].

As a result, there exist infinitely many massive gravity theories corresponding to infinitely many possibilities to choose the potential $U$. One therefore needs a guiding principle to select one particular theory. An example of this is provided by the dRGT potential selected by the requirement that the theory should always propagate $5 \mathrm{DoF}$, for any backgrounds [3]. This potential is expressed in terms of fractional powers of $\hat{S}$,

$$
U=\beta_{0}+\sum_{n=1}^{3} \beta_{k} U_{k}
$$

where $\beta_{0}, \beta_{k}$ are real parameters and

$$
U_{1}=[\hat{\gamma}], \quad U_{2}=\frac{1}{2 !}\left([\hat{\gamma}]^{2}-\left[\hat{\gamma}^{2}\right]\right), \quad U_{3}=\frac{1}{3 !}\left([\hat{\gamma}]^{3}-3[\hat{\gamma}]\left[\hat{\gamma}^{2}\right]+2\left[\hat{\gamma}^{3}\right]\right)
$$

the matrix $\hat{\gamma}$ being determined by the condition $\hat{\gamma}^{2}=\hat{S}$ hence $\hat{\gamma}=\hat{S}^{1 / 2}$.

The OP potential is selected by a different requirement: the theory should imply the linear Hilbert-Lorentz condition as a consequence of the field equations. This potential contains arbitrary real powers of $\hat{S}$. Splitting the inverse metric as

$$
g^{\mu \nu}=f^{\mu \nu}+\chi^{\mu \nu}
$$

one has

$$
S_{\nu}^{\mu}=g^{\mu \sigma} f_{\sigma \nu}=\delta_{\nu}^{\mu}+\chi^{\mu \sigma} f_{\sigma \nu} \equiv \delta_{\nu}^{\mu}+\chi_{\nu}^{\mu},
$$

which can be written as ${ }^{8}$

$$
\hat{S}=\hat{g}^{-1} \hat{f} \equiv \hat{1}+\hat{\chi} .
$$

An arbitrary real power of $\hat{S}$ is defined via the series,

$$
\hat{\Sigma} \equiv \hat{S}^{n}=\hat{1}+n \hat{\chi}+\frac{n(n-1)}{2} \hat{\chi}^{2}+\ldots
$$

Introducing the scalar

$$
\phi=\frac{1}{\sqrt{\operatorname{det}(\hat{S})}}=\left(1+[\hat{\chi}]+\frac{1}{2}\left([\hat{\chi}]^{2}-\left[\hat{\chi}^{2}\right]\right)+\ldots\right)^{-1 / 2},
$$

\footnotetext{
${ }^{8}$ We use the hat is used to denote matrices, e.g. $\hat{S}$, the matrix components being denoted either without hat, $S_{\nu}^{\mu}$, or as $(\hat{S})_{\nu}^{\mu}$.
} 
the OP mass term is given by

$$
U=\mathcal{U}+\lambda_{g}
$$

where the $\hat{S}$-dependent part is

$$
\mathcal{U}=\frac{1}{4 n^{2}} \phi^{s}\left[\hat{S}^{n}\right]=\frac{1}{4 n^{2}}(\operatorname{det}(\hat{S}))^{-s / 2}\left[\hat{S}^{n}\right],
$$

while the constant part is

$$
\lambda_{g}=\frac{n-2 s-2}{2 n^{2}} .
$$

The potential depends on two real parameters $n, s$ (OP use instead $n, p=-(s+1) / n$ ).

The OP theory propagates $6 \mathrm{DoF}$ for generic values of $n$ and $s$. If the parameters belong to the ellipse shown in Fig. 1 below, then the theory has the FP property and propagates only 5 DoF around flat space. For the particular point at the ellipse corresponding to

$$
s=0, \quad n=\frac{1}{2},
$$

the OP potential (2.12) coincides with the dRGT potential (2.5) for

$$
\beta_{0}=-3, \quad \beta_{1}=1, \quad \beta_{2}=\beta_{3}=0 .
$$

The OP theory becomes "ghost-free" in this case, in the sense that it propagates only 5 DoF for generic backgrounds.

The matter term in the action (2.1) can be arbitrary, but we shall be considering just the (boldfaced) cosmological constant,

$$
\mathcal{L}_{\text {matter }}=-\Lambda \text {. }
$$

\section{$2.1 \quad$ Field equations}

Let us vary the two metrics, $g_{\mu \nu} \rightarrow g_{\mu \nu}+\delta g_{\mu \nu}$ and $f_{\mu \nu} \rightarrow f_{\mu \nu}+\delta f_{\mu \nu}$ (the metric $f_{\mu \nu}$ can be varied even though it is non-dynamical, in order to obtain identities similar to the Bianchi identity). The potential (2.12) then receives the variation

$$
\delta(U \sqrt{-g})=\frac{1}{2}\left(\sqrt{-g} X^{\mu \nu} \delta g_{\mu \nu}-\sqrt{-f} Y^{\mu \nu} \delta f_{\mu \nu}\right),
$$

where $X^{\mu \nu}=X_{\alpha}^{\mu} g^{\alpha \nu}$ and $Y^{\mu \nu}=Y_{\alpha}^{\mu} f^{\alpha \nu}$ with

$$
\begin{aligned}
X_{\nu}^{\mu} & =\frac{1}{2 n^{2}} \phi^{s}\left(n \Sigma_{\nu}^{\mu}-\frac{s+1}{2} \Sigma_{\alpha}^{\alpha}{ }_{\alpha}{ }_{\nu}^{\mu}\right), \\
Y_{\nu}^{\mu} & =\frac{1}{2 n^{2}} \phi^{s+1}\left(n \Sigma^{\mu}{ }_{\nu}-\frac{s}{2} \Sigma^{\alpha}{ }_{\alpha} \delta^{\mu}{ }_{\nu}\right),
\end{aligned}
$$

where $\Sigma^{\mu}{ }_{\nu}=\left(\hat{S}^{n}\right)_{\nu}^{\mu}$. Consider an infinitesimal diffeomorphism generated by a vector field $\xi^{\mu}$. It induces the variations of both metrics,

$$
\delta g_{\mu \nu}=\stackrel{(g)}{\nabla}_{(\mu} \stackrel{(g)}{\xi}_{\nu)}, \quad \delta f_{\mu \nu}=\stackrel{(f)}{\nabla}_{(\mu} \stackrel{(f)}{\xi}_{\nu)},
$$


where $\stackrel{(g)}{\xi}_{\mu}=g_{\mu \sigma} \xi^{\sigma}$ and $\stackrel{(f)}{\xi}_{\mu}=f_{\mu \sigma} \xi^{\sigma}$ while $\stackrel{(g)}{\nabla}$ and $\stackrel{(f)}{\nabla}$ are the covariant derivatives with respect to the g-metric and f-metric, respectively. Inserting this to (2.18), integrating over the manifold, dropping the boundary term and using the fact that $U$ is a scalar and hence its integral does not change under diffeomorphisms, gives the identity

$$
\sqrt{-g} \stackrel{(g)}{\nabla}{ }_{\mu} X_{\nu}^{\mu}=\sqrt{-f} \stackrel{(f)}{\nabla}_{\mu} Y_{\nu}^{\mu}
$$

Let us now vary the whole action only with respect to $g_{\mu \nu}$. Setting the variation to zero, yields the equations

$$
G_{\nu}^{\mu}+m^{2} \lambda_{g} \delta_{\nu}^{\mu}=m^{2} X_{\nu}^{\mu}+T_{\nu}^{(\mathrm{m}) \mu}
$$

where $T_{\mu \nu}^{(\mathrm{m})}$ is obtained by varying $\mathcal{L}_{\text {matter }}$. If the latter is given by $(2.17)$ then

$$
T_{\nu}^{(\mathrm{m}) \mu}=-\Lambda \delta_{\nu}^{\mu}
$$

\subsection{Subsidiary conditions}

In view of the Bianchi identities $\stackrel{(g)}{\nabla}_{\mu} G^{\mu}{ }_{\nu}=0$ and owing to the matter conservation condition $\stackrel{(g)}{\nabla} T_{\mu}^{(\mathrm{m}) \mu}=0$, equations (2.22) imply that

$$
\stackrel{(g)}{\nabla}_{\mu} X_{\nu}^{\mu}=0
$$

This in turn implies, in view of the identity (2.21), that

$$
\stackrel{(f)}{\nabla_{\mu}} Y_{\nu}^{\mu}=0
$$

Now, according to (2.8), one has $S_{\nu}^{\mu}=\delta^{\mu}{ }_{\nu}+\chi_{\nu}^{\mu}$, where $\chi_{\nu}^{\mu}$ vanishes if $g_{\mu \nu}=f_{\mu \nu}$. The weighted power of this matrix can be represented similarly,

$$
\phi^{s+1}\left(S^{n}\right)^{\mu}{ }_{\nu}=\delta^{\mu}{ }_{\nu}+\Psi_{\nu}^{\mu}
$$

where $\Psi^{\mu}{ }_{\nu}$ vanishes when the two metrics coincide. This relation is in fact equivalent to the one in (1.11), assuming that the indices are moved with the f-metric so that

$$
\left(\left(\hat{g}^{-1}\right)^{n}\right)^{\mu \nu}=\left(\hat{S}^{n}\right)_{\sigma}^{\mu} f^{\sigma \nu}, \quad \Psi^{\mu \nu}=\Psi_{\sigma}^{\mu} f^{\sigma \nu} .
$$

Using (2.26) reduces $(2.25)$ to

$$
\stackrel{(f)}{\nabla}_{\mu}\left(\Psi_{\nu}^{\mu}-\frac{s}{2 n}\left(\Psi_{\alpha}^{\alpha}\right) \delta_{\nu}^{\mu}\right)=0
$$

If the reference metric is chosen to be flat Minkowski, $f_{\mu \nu}=\eta_{\mu \nu}$, then the derivatives $\stackrel{(f)}{\nabla}$ m become ordinary partial derivatives and (2.28) reduces to the Lorentz-Hilbert condition for the $\Psi$-field,

$$
\partial_{\mu} \Psi_{\nu}^{\mu}+q \partial_{\nu} \Psi_{\alpha}^{\alpha}=0
$$


with $q=-s /(2 n)$. This explains the OP's trick - the linear in $\Psi_{\nu}^{\mu}$ subsidiary condition (2.29) indeed follows from the non-linear field equations (2.22). This explains also why this condition is not manifestly covariant - the field equations (2.22) are covariant if only both metrics are allowed to simultaneously transform, but the covariance is lost as soon as the metric $f_{\mu \nu}$ is fixed (unitary gauge).

Even though the OP theory is formulated entirely in terms of the metrics $g_{\mu \nu}$ and $f_{\mu \nu}$, according to the OP's philosophy, the metric $g_{\mu \nu}$ is only a secondary object. The primary object is supposed to be the graviton field $\Psi_{\nu}^{\mu}$ determining the metric $g_{\mu \nu}$ via (2.26). Setting the philosophy aside, the mathematical statement is that in the OP theory there exists the invertible non-linear transformation (2.26) expressing the metric $g_{\mu \nu}$ in terms of $\Psi^{\mu \nu}$ such that the condition $\partial_{\mu} Y_{\nu}^{\mu}=0$ becomes linear in $\Psi^{\mu \nu}$.

One should stress at the same time that the tensor $Y_{\nu}^{\mu}$ can be defined via (2.18) in any bimetric theory, for any choice of the mass term $U$. The condition (2.25) will always hold on-shell, and setting $f_{\mu \nu}=\eta_{\mu \nu}$ one always obtains $\partial_{\mu} Y_{\nu}^{\mu}=0$. However, $Y_{\nu}^{\mu}$ will in general contain non-linear terms that cannot be absorbed by redefining the variables via (2.26), hence conditions $\partial_{\mu} Y_{\nu}^{\mu}=0$ will not have the Lorentz-Hilbert form needed for the "spin limitation".

For example, in the dRGT theory with the potential (2.5) one has

$$
Y_{\nu}^{\mu}=-\frac{1}{\operatorname{det}(\hat{\gamma})}\left\{\left(\beta_{1}+\beta_{2} U_{1}+\beta_{3} U_{2}\right) \gamma_{\nu}^{\mu}-\left(\beta_{2}+\beta_{3} U_{1}\right)\left(\hat{\gamma}^{2}\right)_{\nu}^{\mu}+\beta_{3}\left(\hat{\gamma}^{3}\right)_{\nu}^{\mu}\right\} \text {. }
$$

If $\beta_{2}=\beta_{3}=0$ then the conservation condition $\partial_{\mu} Y_{\nu}^{\mu}=0$ reduces to

$$
\partial_{\mu}\left(\frac{1}{\operatorname{det}(\hat{\gamma})} \gamma_{\nu}^{\mu}\right)=0
$$

which has the form (2.26) and can be linearized by setting

$$
\frac{1}{\operatorname{det}(\hat{\gamma})} \gamma^{\mu}{ }_{\nu}=\delta^{\mu}{ }_{\nu}+\Psi_{\nu}^{\mu}
$$

which yields $\partial_{\mu} \Psi_{\nu}^{\mu}=0$. However, the same trick does not work for generic values of $\beta_{k}$. For example, if $\beta_{1}=\beta_{3}=0$ and $\beta_{2} \neq 0$ then one obtains

$$
\partial_{\mu}\left(\frac{1}{\operatorname{det}(\hat{\gamma})}\left([\hat{\gamma}] \gamma_{\nu}^{\mu}-\left(\hat{\gamma}^{2}\right)_{\nu}^{\mu}\right)\right)=0
$$

which cannot be linearized by applying (2.26). Of course, this can be linearized by a different transformation. However, within the OP approach, the transformation should be the same as the one OP used to derive the Einstein-Hilbert kinetic term starting from the linear theory, hence it must have the form (2.26), which is equivalent to (1.11).

Summarizing, the OP potential is adjusted in such a way that the tensor $Y_{\nu}^{\mu}$ has the structure $Y_{\nu}^{\mu}=D_{\nu}^{\mu}+$ const. $\times[\hat{D}] \delta_{\nu}^{\mu}$ where $D_{\nu}^{\mu}$ is the weighted power of $g_{\mu \nu}$. Changing the variables via $D^{\mu}{ }_{\nu}=\delta^{\mu}{ }_{\nu}+\Psi_{\nu}^{\mu}$, the one-shell condition $\partial_{\mu} Y_{\nu}^{\mu}=0$ assumes the linear form $\partial_{\mu} \Psi^{\mu}{ }_{\nu}+q \partial_{\nu} \Psi_{\alpha}^{\alpha}=0$. According to OP, this property is very important, since the linear condition for $\Psi^{\mu \nu}$ restricts the spin of states in the theory. To understand what this property gives in practical terms, we shall now study the phenomenology of the theory. 


\section{Solutions with proportional metrics}

We shall first consider the simplest solutions for which the reference metric $f_{\mu \nu}$ is not fixed once and forever but related to the physical metric via

$$
f_{\mu \nu}=\xi^{2} g_{\mu \nu}
$$

with constant $\xi$. This implies that

$$
S^{\mu}{ }_{\nu}=\xi^{2} \delta^{\mu}{ }_{\nu} \quad \Rightarrow \quad X^{\mu}{ }_{\nu}=\lambda_{g} \xi^{2(n-2 s)} \delta^{\mu}{ }_{\nu},
$$

and the field equations (2.22) reduce to

$$
G_{\mu \nu}+\Lambda g_{\mu \nu}=0
$$

with

$$
\Lambda=\Lambda+m^{2} \lambda_{g}\left[1-\xi^{2(n-2 s)}\right] .
$$

It follows that the solution is an Einstein space with $R_{\mu \nu}=\Lambda g_{\mu \nu}$, for example the de Sitter space. If the parameters are adjusted such that $\Lambda=0$, then the flat space will be a solution. We emphasize once again that to different solutions there correspond different reference metrics defined via (3.1).

Let us now analyze the stability of such solutions. To this end, we consider small perturbations of the g-metric without changing the reference metric,

$$
g_{\mu \nu} \rightarrow g_{\mu \nu}+h_{\mu \nu}, \quad f_{\mu \nu} \rightarrow f_{\mu \nu},
$$

hence

$$
g^{\mu \nu} \rightarrow g^{\mu \nu}-h^{\mu \nu}+\ldots,
$$

with $h^{\mu \nu}=g^{\mu \alpha} g^{\nu \beta} h_{\alpha \beta}$. Let us consider the linear in perturbations part of the field equations,

$$
\delta G_{\mu \nu}+\left(\boldsymbol{\Lambda}+m^{2} \lambda_{g}\right) \delta g_{\mu \nu}=m^{2} \delta X_{\mu \nu},
$$

where

$$
\delta X_{\mu \nu}=\delta\left(g_{\mu \sigma} X_{\nu}^{\sigma}\right)=h_{\mu \sigma} X_{\nu}^{\sigma}+g_{\mu \sigma} \delta X_{\nu}^{\sigma}=\lambda_{g} \xi^{2(n-2 s)} h_{\mu \nu}+g_{\mu \sigma} \delta X_{\nu}^{\sigma} .
$$

To calculate the variation $\delta X^{\sigma}{ }_{\nu}$, we notice that the tensor $X^{\mu}{ }_{\nu}$ defined by (2.19) contains powers of the tensor $S^{\mu}{ }_{\nu}=g^{\mu \sigma} f_{\sigma \nu}$, whose variation is

$$
\delta S^{\mu}{ }_{\nu}=\delta g^{\mu \sigma} f_{\sigma \nu}=-h^{\mu \sigma} f_{\sigma \nu}=-h^{\mu}{ }_{\sigma} S_{\nu}^{\sigma}=-\xi^{2} h^{\mu}{ }_{\nu} .
$$

The background tensor $S^{\mu}{ }_{\nu}=\xi^{2} \delta_{\nu}^{\mu}$ is proportional to the unit tensor, hence it commutes with $\delta S^{\mu}{ }_{\nu}$, which implies that

$$
\delta \Sigma_{\nu}^{\mu}=\delta\left(S^{n}\right)^{\mu}{ }_{\nu}=n \delta S^{\mu}{ }_{\sigma}\left(S^{n-1}\right)^{\sigma}{ }_{\nu}=-n h^{\mu}{ }_{\sigma}\left(S^{n}\right)^{\sigma}{ }_{\nu}=-n \xi^{2 n} h^{\mu}{ }_{\nu} .
$$

It follows also that

$$
\delta(\operatorname{det} \hat{S})=\delta\left(\operatorname{det} \hat{g}^{-1}\right) \operatorname{det} \hat{f}=-\operatorname{det}(\hat{S}) h
$$


with $h=h^{\mu}{ }_{\mu}$ and hence

$$
\left.\delta\left(\phi^{s}\right)=\delta(\operatorname{det} \hat{S})^{-s / 2}\right)=\frac{s}{2} \phi^{s} h=\frac{s}{2} \xi^{-4 s} h .
$$

As a result,

$$
\begin{aligned}
\delta X_{\nu}^{\mu} & =\frac{s}{2} h X_{\nu}^{\mu}-\frac{1}{2} \xi^{2 n-4 s}\left(n h^{\mu}{ }_{\nu}-\frac{s+1}{2} h \delta^{\mu}{ }_{\nu}\right) \\
& =\xi^{2 n-4 s}\left(-\frac{1}{2} h^{\mu}{ }_{\nu}+\left[\frac{s}{2} \lambda_{g}+\frac{s+1}{4 n}\right] h \delta^{\mu}{ }_{\nu}\right)
\end{aligned}
$$

and the perturbation equations (3.7) assume the form

$$
E_{\mu \nu} \equiv \delta G_{\mu \nu}+\Lambda h_{\mu \nu}+\frac{M^{2}}{2}\left(h_{\mu \nu}-\lambda h g_{\mu \nu}\right)=0
$$

with $\Lambda$ defined by (3.4) and with

$$
M^{2}=m^{2} \xi^{2 n-4 s}
$$

while

$$
\lambda=1+\frac{1-(n-2 s-1)^{2}-3 n^{2}}{4 n^{2}} \equiv 1+\zeta .
$$

For $\lambda=1$ Eqs.(3.14) reduce to those studied by Higuchi to describe massive gravitons in de Sitter space [25], the parameter $M$ then determines the graviton mass. The equations show the FP property in this case - they propagate only 5 DoF. However, for $\lambda \neq 1$ the number of DoF is 6 . Let us remind the corresponding counting argument.

There are 10 equations in (3.14), where one has

$$
\begin{aligned}
\delta G_{\mu \nu}= & \frac{1}{2}\left(\nabla^{\sigma} \nabla_{\mu} h_{\sigma \nu}+\nabla^{\sigma} \nabla_{\nu} h_{\sigma \mu}-\square h_{\mu \nu}-\nabla_{\mu} \nabla_{\nu} h-R h_{\mu \nu}\right) \\
& +\frac{1}{2} g_{\mu \nu}\left(\square h-\nabla^{\alpha} \nabla^{\beta} h_{\alpha \beta}+R^{\alpha \beta} h_{\alpha \beta}\right) .
\end{aligned}
$$

For $R_{\mu \nu}=\Lambda g_{\mu \nu}$ and $R=4 \Lambda$ there is the identity relation $\nabla^{\mu}\left(\delta G_{\mu \nu}+\Lambda h_{\mu \nu}\right)=0$, hence taking the divergence of (3.14) yields four constraints

$$
\frac{M^{2}}{2}\left(\nabla^{\mu} h_{\mu \nu}-\lambda \nabla_{\nu} h\right)=0
$$

from which $\nabla^{\mu} h_{\mu \nu}=\lambda \nabla_{\nu} h$. Using these relations, Eqs.(3.14) reduce to

$$
\begin{aligned}
-\square h_{\mu \nu}+(2 \lambda-1) \nabla_{\mu \nu} h-2 R_{\mu \alpha \nu \beta} h^{\alpha \beta} & +[(1-\lambda) \square h+\Lambda h] g_{\mu \nu} \\
& +M^{2}\left[h_{\mu \nu}-\lambda h g_{\mu \nu}\right]=0,
\end{aligned}
$$

and taking the trace one obtains

$$
2(1-\lambda) \square h+\left[2 \Lambda+(1-4 \lambda) M^{2}\right] h=0 .
$$


If $\lambda=1$ then this yields

$$
\left(2 \Lambda-3 M^{2}\right) h=0,
$$

implying the fifth constraint, $h=0$ (unless in the partially massless limit $2 \Lambda=3 M^{2}$ ). Therefore, the number of DoF is the number of components of $h_{\mu \nu}$ minus the number of constraints in (3.18),(3.20), which gives $10-5=5$. This corresponds to the Fierz-Pauli theory.

If $\lambda \neq 1$ then the FP property is lost, because there is the non-trivial kinetic term in (3.20), hence the trace $h$ becomes a dynamical mode, so that there are 6 DoF.

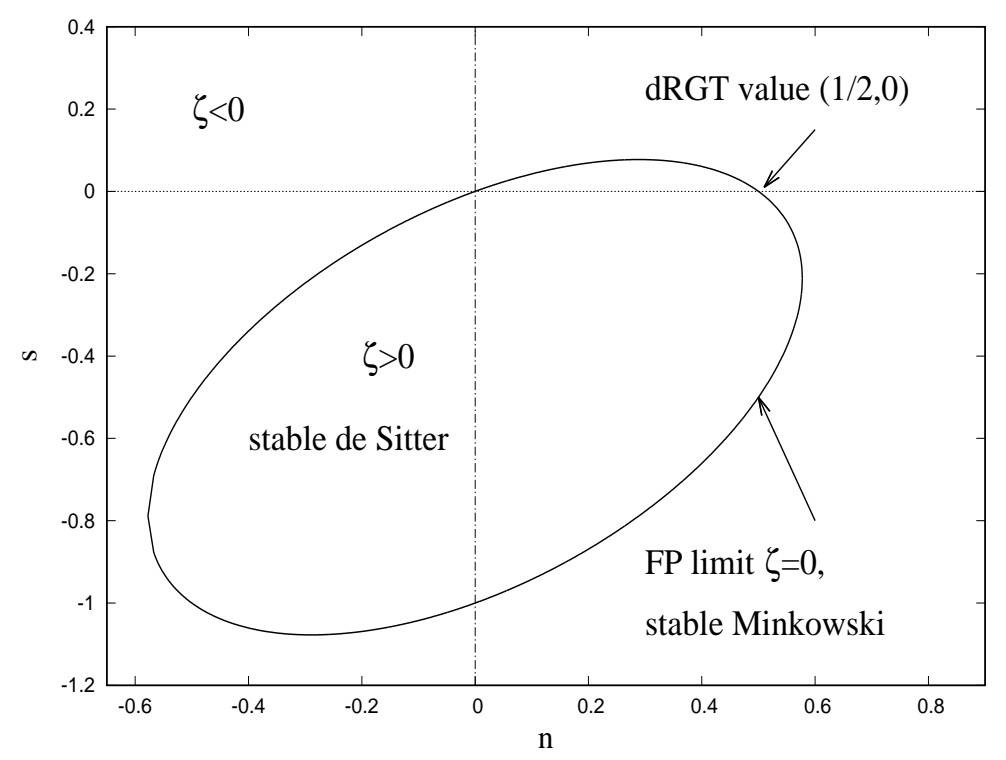

Figure 1. The FP subset of the OP theory.

Therefore, the OP theory respects the FP property if the parameter $\lambda$ defined by (3.16) is equal to one, hence if

$$
(n-2 s-1)^{2}+3 n^{2}=1 .
$$

This defines an ellipse in the $n s$-plane shown in Fig.1. Points of this ellipse correspond to the special case of the OP theory in which the FP property is respected and there are only $5 \mathrm{DoF}$ around the de Sitter (or flat for $\Lambda=0$ ) space. Points not belonging to the ellipse correspond to the generic OP theory with 6 DoF.

If $\Lambda=0$ and the background geometry is flat, then among the 6 DoF there is ghost - a mode with a negative kinetic energy. One might think that all of the OP theories with 6 DoF are unphysical, since flat space is unstable in such theories. However, as we shall now see, the whole interior of the ellipse corresponds to theories in which the de Sitter space is stable at the level of liner perturbations. 


\section{Stability conditions}

Let us assume the background metric $g_{\mu \nu}$ to be of the Friedmann-Lemaitre-RobertsonWalker (FLRW) type,

$$
d s_{g}^{2}=-N^{2}(t) d t^{2}+a^{2}(t) \delta_{i j} d x^{i} d x^{j}=\frac{1}{\xi^{2}} d s_{f}^{2}
$$

which fulfills background equations (3.3) with $\Lambda \equiv 3 H^{2}>0$,

$$
\frac{\dot{a}^{2}}{N^{2} a^{2}}=H^{2}
$$

This describes the de Sitter space expressed in the spatially flat slicing.

Perturbing the solution,

$$
g_{\mu \nu} \rightarrow g_{\mu \nu}+h_{\mu \nu}, \quad f_{\mu \nu} \rightarrow f_{\mu \nu}
$$

the perturbations can be decomposed into the scalar, vector, and tensor parts via

$$
\begin{aligned}
h_{00} & =-N^{2} \mathbf{S}_{3}, \\
h_{0 i} & =N a\left(\partial_{i} \mathbf{S}_{4}+\mathbf{W}_{i}\right), \\
h_{i k} & =a^{2}\left(\mathbf{S}_{1} \delta_{i k}+\partial_{i k}^{2} \mathbf{S}_{2}+\partial_{i} \mathbf{V}_{k}+\partial_{k} \mathbf{V}_{i}+\mathbf{D}_{i k}\right),
\end{aligned}
$$

where

$$
\sum_{k} \partial_{k} \mathbf{V}_{k}=\sum_{k} \partial_{k} \mathbf{W}_{k}=0, \quad \sum_{k} \partial_{k} \mathbf{D}_{k i}=0, \quad \sum_{k} \mathbf{D}_{k k}=0 .
$$

The spatial dependence of the modes is given by the plane waves $\exp (i \mathbf{p x})=\exp (i p z)$, where the wave vector can be oriented along the 3-rd (z) axis. The amplitudes $\mathbf{S}_{4}$ and $\mathbf{V}_{k}$ have dimension of length, while $\mathbf{S}_{2}$ has dimension of length squared. To pass to dimensionless quantities, we introduce a mass scale $\mu$ and set

$$
\mathbf{S}_{1}=S_{1}(t) e^{i p z}, \quad \mathbf{S}_{2}=\frac{1}{\mu^{2}} S_{2}(t) e^{i p z}, \quad \mathbf{S}_{3}=S_{3}(t) e^{i p z}, \quad \mathbf{S}_{4}=\frac{1}{\mu} S_{4}(t) e^{i p z},
$$

the vector amplitudes are chosen as

$$
\mathbf{V}_{k}=\frac{1}{\mu}\left[V_{1}(t), V_{2}(t), 0\right] e^{i p z}, \quad \mathbf{W}_{k}=\left[W_{1}(t), W_{2}(t), 0\right] e^{i p z}
$$

while for the tensor modes the only non-trivial components of $\mathbf{D}_{i k}$ are

$$
\mathbf{D}_{11}=-\mathbf{D}_{22}=D_{1}(t) e^{i p z}, \quad \mathbf{D}_{12}=\mathbf{D}_{21}=D_{2}(t) e^{i p z}
$$

The mass scale $\mu$ can be, for example, the Planck mass $M_{\mathrm{Pl}}$, or the Hubble rate $H$, or the graviton mass $M$. However, we prefer not to specify it to be able to consider the limits such as $H \rightarrow 0$ or $M \rightarrow 0$. 
Inserting everything into the perturbation equations $E_{i k}=0$ (3.14), they split into three independent groups for the scalar, vector, and tensor modes. These equations determine the effective action, which is the sum of three independent terms,

$$
I=I_{\mathrm{T}}+I_{\mathrm{V}}+I_{\mathrm{S}}=\frac{M_{\mathrm{Pl}}^{2}}{4} \int N a^{3} \bar{h}^{\mu \nu} E_{\mu \nu} d t d^{3} x,
$$

where the bar denotes complex conjugation. One obtains in the tensor sector

$$
I_{\mathrm{T}}=\frac{M_{\mathrm{Pl}}^{2}}{4} \int N a^{3}\left(\frac{1}{N^{2}}\left(\dot{D}_{1}^{2}+\dot{D}_{2}^{2}\right)-\left[M^{2}+\frac{p^{2}}{a^{2}}\right]\left(D_{1}^{2}+D_{2}^{2}\right)\right) d t d^{3} x .
$$

Inspecting the equations in the vector sector one finds that the two amplitudes $W_{1}$ and $W_{2}$ can be expressed in therms of $V_{1}$ and $V_{2}$, the latter being governed by the action

$$
I_{\mathrm{V}}=\frac{M_{\mathrm{Pl}}^{2}}{4 \mu^{2}} M^{2} \int N a^{3}\left(\frac{p^{2}}{N^{2}\left(p^{2} / a^{2}+M^{2}\right)}\left(\dot{V}_{1}^{2}+\dot{V}_{2}^{2}\right)-p^{2}\left(V_{1}^{2}+V_{2}^{2}\right)\right) d t d^{3} x .
$$

Neither tensor nor vector modes are sensitive to the value of the parameter $\zeta$ describing the deviation from the FP limit. The vector modes become non-dynamical when the mass $M$ tends to zero.

In the scalar sector, the amplitudes $S_{3}$ and $S_{4}$ can be expressed in terms of $S_{1}$ and $S_{2}$, the effective action for the latter being

$$
I_{\mathrm{S}}^{(\zeta)}=\frac{M_{\mathrm{Pl}}^{2}}{4} M^{2} \int N a^{3} \sum_{a, b=1,2}\left(\frac{1}{N^{2}} K_{a b} \dot{S}_{a} \dot{S}_{b}+\frac{\mathcal{Q}}{N} \epsilon_{a b} \dot{S}_{a} S_{b}-U_{a b} S_{a} S_{b}\right) d t d^{3} x .
$$

Here the kinetic matrix has components

$$
K_{11}=\zeta \frac{3 a^{2} M^{2}+4 p^{2}}{X}, \quad K_{22}=\frac{2 a^{2} H^{2} p^{4}}{\mu^{4} X}, \quad K_{12}=K_{21}=-\zeta \frac{a^{2} M^{2} p^{2}}{\mu^{2} X},
$$

with

$$
X=8 H^{2} p^{2}+M^{2} a^{2}\left(6 H^{2}-\zeta M^{2}\right),
$$

and one has

$$
\mathcal{Q}=-2(1+2 \zeta) \frac{H p^{4}}{\mu^{2} X}, \quad \epsilon_{a b}=-\epsilon_{b a}, \quad \epsilon_{12}=1 .
$$

Components of the potential matrix have more complex structure,

$$
\begin{array}{r}
U_{11}=\frac{1}{2 a^{2} X^{2}}\left\{-32 H^{2} p^{6}+4 a^{2}\left[48 H^{4}-6 M^{2}(2 \zeta+5) H^{2}+\zeta M^{4}\right] p^{4}\right. \\
+2 M^{2} a^{4}\left[144 H^{4}+6\left(4 \xi^{2}-11 \xi-12\right) M^{2} H^{2}+\zeta(5 \zeta+6) M^{4}\right] p^{2} \\
\left.+3 M^{4} a^{6}\left(6 H^{2}-\zeta M^{2}\right)\left(6 H^{2}-(3+4 \zeta) M^{2}\right)\right\}, \\
U_{22}=\frac{p^{4}}{2 \mu^{4} X^{2}}\left\{-64 \zeta H^{4} p^{4}-8 a^{2} H^{2} M^{2}\left[(2 \zeta-10) H^{2}+\left(1+2 \zeta-\zeta^{2}\right) M^{2}\right] p^{2}\right. \\
\left.+M^{4} a^{4}\left(6 H^{2}-(1+2 \xi) M^{2}\right)\left(6 H^{2}-\zeta M^{2}\right)\right\}, \\
U_{12}=U_{21}=\frac{p^{2}}{2 \mu^{2} X^{2}}\left\{16 H^{2}\left[(6 \zeta-1) H^{2}+(1+\zeta) M^{2}\right] p^{4}\right. \\
+2 a^{2} M^{2}\left[6(2 \zeta-7) H^{4}-\left(10 \zeta^{2}-17 \zeta-18\right) M^{2} H^{2}-\zeta(1+\zeta) M^{4}\right] p^{2} \\
\left.-M^{4} a^{4}\left(6 H^{2}-\zeta M^{2}\right)\left(6 H^{2}-(3+4 \zeta) M^{2}\right)\right\} .
\end{array}
$$


We notice that the scalars become non-dynamical in the $M \rightarrow 0$ limit.

Let us analyze the positivity of the kinetic matrix $K_{a b}$. This eigenvalues $\lambda_{1}$ and $\lambda_{2}$ of this matrix will be positive definite if the trace $\operatorname{tr}\left(K_{a b}\right)=\lambda_{1}+\lambda_{2}$ and the determinant $\operatorname{det}\left(K_{a b}\right)=\lambda_{1} \lambda_{2}$ are both positive. One has

$$
\begin{aligned}
\operatorname{tr}\left(K_{a b}\right) & =\frac{1}{\mu^{4} X}\left(2 H^{2} a^{2} p^{4}+4 \zeta \mu^{4} p^{2}+3 \zeta a^{2} \mu^{4} M^{2}\right) \stackrel{p \rightarrow \infty}{\longrightarrow} \frac{a^{2} p^{2}}{4 \mu^{4}}+\mathcal{O}(1), \\
\operatorname{det}\left(K_{a b}\right) & =\frac{1}{\mu^{4} X} \zeta a^{2} p^{4} \stackrel{p \rightarrow \infty}{\longrightarrow} \frac{\zeta a^{2}}{8 \mu^{4} H^{2}} p^{2}+\mathcal{O}(1) .
\end{aligned}
$$

Therefore, as long as $\zeta>0$, the kinetic matrix is always positive-definite in the UV limit $p \rightarrow \infty$, and it will be positive definite for any momenta if

$$
6 H^{2}>\zeta M^{2}
$$

since $X>0$ in this case. This conclusion applies only when the Hubble rate of the background metric is non-zero, $H \neq 0$.

If $H=0$ then the background metric is flat and one can set $a=1$, hence (4.14) yields $X=-\zeta M^{4}$. Eq.(4.17) then gives

$$
\operatorname{tr}\left(K_{a b}\right)=-\frac{4 p^{2}+3 M^{2}}{M^{4}}<0, \quad \operatorname{det}\left(K_{a b}\right)=-\frac{p^{4}}{\mu^{4} M^{4}}<0,
$$

hence one of the two eigenvalues $\lambda_{1}$ and $\lambda_{2}$ is always negative.

As a result, there is ghost around flat space if $\zeta \neq 0$. This fact is of course wellknown. What is new is that the ghost becomes a benign mode in the de Sitter space if $\zeta>0$.

One may wonder if there are gradient instabilities in the system. The sound speed $C_{\mathrm{S}}$ is determined by the algebraic equation

$$
\operatorname{det}\left(\frac{p^{2} C_{\mathrm{S}}^{2}}{a^{2}} K_{a b}+i \frac{p C_{\mathrm{S}}}{a} \mathcal{Q} \epsilon_{a b}-U_{a b}\right)=0 .
$$

This gives two different values for $C_{\mathrm{S}}^{2}$ determining the speed of the scalar component of the massive graviton and that of the 6-th mode. Both have the same UV limit,

$$
\lim _{p \rightarrow \infty} C_{\mathrm{S}}^{2}=1 .
$$

Since $C_{\mathrm{S}}^{2}>0$, there are no gradient instabilities in this limit. This conclusion applies both for the de Sitter space $(H \neq 0)$ and for Minkowski space $(H=0)$.

\subsection{The FP limit}

Let us finally see what happens when $\zeta \rightarrow 0$. The only non-vanishing component of the kinetic matrix $K_{a b}$ in this limit is $K_{22}$, hence the amplitude $S_{1}$ becomes non-dynamical and can be algebraically expressed in terms of $S_{2}$. Injecting this expression back to the action yields

$$
I_{\mathrm{S}}^{(\zeta=0)}=\frac{3 M_{\mathrm{Pl}}^{2}}{4 \mu^{4}} M^{2}\left(M^{2}-2 H^{2}\right) \int N a^{3}\left(\frac{1}{N^{2}} K \dot{S}_{2}^{2}-U S_{2}^{2}\right) d t d^{3} x
$$


with

$$
K=\frac{a^{4} p^{4}}{Y}, \quad U=a^{2} p^{4} \frac{16 H^{2} a^{2} p^{4}+\left(p^{2}+a^{2} M^{2}\right) Y}{Y^{2}},
$$

where $Y=4 p^{4}+3 a^{2}\left(M^{2}-2 H^{2}\right)\left(4 p^{2}+3 M^{2} a^{2}\right)$. We see that the scalar mode becomes non-dynamical either for $M=0$ (massless limit) or for $M^{2}=2 H^{2}$ (partially massless limit). The kinetic therm is always positive if $M^{2}>2 H^{2}$ but for $M^{2}<2 H^{2}$ it becomes negative and the scalar mode becomes the (Higuchi) ghost. The speed of sound is equal to one in the UV limit. Sending $H \rightarrow 0$, one can see that the flat space is stable in this case.

\subsection{Generic massive gravity}

The above results are actually quite general and apply not only in the OP theory but also in the generic bimetric theory (2.1). Specifically, introducing $H^{\mu}{ }_{\nu}=\delta^{\mu}{ }_{\nu}-S^{\mu}{ }_{\nu}$ with $S_{\nu}^{\mu}=g^{\mu \sigma} f_{\sigma \nu}$, the potential $U$ in (2.1) can be expanded as

$$
U=a_{0}+a_{1}[\hat{H}]+a_{2}\left[\hat{H}^{2}\right]+a_{3}[\hat{H}]^{2}+\ldots
$$

If the matter term in (2.1) is given by (2.17), then the field equations read

$$
G_{\mu \nu}+\Lambda g_{\mu \nu}+m^{2}\left(2 a_{1} f_{\mu \nu}+4 a_{2} f_{\mu \sigma} H_{\nu}^{\sigma}+4 a_{3}[\hat{H}] f_{\mu \nu}+\left(a_{0}+a_{1}[\hat{H}]\right) g_{\mu \nu}+\ldots\right)=0 .
$$

If $f_{\mu \nu}=g_{\mu \nu}$ then $H_{\nu}^{\mu}=0$ and the equations reduce to

$$
G_{\mu \nu}+\Lambda g_{\mu \nu}=0 \quad \text { with } \quad \Lambda=\Lambda+m^{2}\left(a_{0}+2 a_{1}\right) .
$$

Therefore, if the reference metric is chosen to be de Sitter, then the theory admits a solution for which the physical metric is also de Sitter. Consider perturbations $g_{\mu \nu}=f_{\mu \nu} \rightarrow f_{\mu \nu}+h_{\mu \nu}$ with fixed $f_{\mu \nu}$. Linearizing Eqs.(4.25) with respect to $h_{\mu \nu}$ then yields precisely the Higuchi equations (3.14) with

$$
M^{2}=8 m^{2} a_{2}, \quad \lambda=1+\zeta=-\frac{a_{3}}{a_{2}} .
$$

Therefore, the above analysis directly applies and one can say at once that the flat space (obtained if $\Lambda=0$ ) is stable if $a_{2}+a_{3}=0$, while the de Sitter space is stable if $\lambda>1$.

\section{More general cosmologies}

Let us now study more general solutions of the OP theory. We shall be considering FLRW cosmologies described by

$$
\begin{aligned}
& d s_{g}^{2}=-N^{2}(t) d t^{2}+a^{2}(t) \Omega_{i j} d x^{i} d x^{j}, \\
& d s_{f}^{2}=-N_{f}^{2}(t) d t^{2}+a_{f}^{2}(t) \Omega_{i j} d x^{i} d x^{j},
\end{aligned}
$$

where $\Omega_{i j} d x^{i} d x^{j}$ is the metric of the maximally symmetric 3 -space with constant curvature $K=0, \pm 1$. We can assume without loss of generality that the functions 
$N, N_{f}, a, a_{f}$ are positive. The time reparameterization freedom can be used to impose one gauge condition, for example, one can set $N=1$, or $N_{f}=1$, or $a=t$. Denoting

$$
\frac{a_{f}}{a} \equiv \xi, \quad \frac{N_{f}}{N} \equiv c \xi,
$$

and injecting everything to (2.2) and (2.11) yields

$$
S^{\alpha}{ }_{\beta}=\xi^{2} \operatorname{diag}\left[c^{2}, 1,1,1\right], \quad \phi=\left(c \xi^{4}\right)^{-1} .
$$

The function $c$ is the speed of light measured with respect to the reference metric.

\subsection{Stability conditions}

Before we study solutions of the form (5.1), let us describe their stability conditions. These conditions are derived in the Appendix ${ }^{9}$, without imposing the background field equations and only assuming that $\dot{a} \neq 0$. It turns out that backgrounds (5.1) may in general accommodate ghost and gradient instabilities. Ghosts (excitations with a negative kinetic energy) will be absent in the UV limit, for momenta much larger than the Hubble parameter,

if the following condition holds:

$$
p \gg H=\frac{\dot{a}}{N a}
$$

$$
\zeta(c) \equiv-\frac{(2 n-s-1)(2 n-s) c^{2 n}+3 s(s+1)}{4 n^{2}}>0 .
$$

However, soft ghosts with a wavelength of the order or larger than the cosmological horizon may still be present. Notice that if $c \rightarrow 1$ then $\zeta(c)$ reduces to $\zeta$ defined by (3.16).

The gradient instability is characterized by an imaginary sound speed. The sound speed $C_{\mathrm{S}}$ is determined by the algebraic equation (again assuming the UV limit (5.4))

$$
2 C_{\mathrm{V}}^{2} \zeta(c) C_{\mathrm{S}}^{4}+\left[C_{\mathrm{V}}^{4}\left(4 Z \zeta(c)-\omega^{2}\right)+c^{2}\right] C_{\mathrm{S}}^{2}+2 c^{2} C_{\mathrm{V}}^{2} Z=0,
$$

where

$$
\begin{aligned}
\omega & =-\frac{1+c^{2}}{2 C_{\mathrm{V}}^{2}}+\frac{2 s+1}{2 n}\left(1+c^{2 n}\right)-\frac{s(s+1)}{2 n^{2}}\left(3+c^{2 n}\right), \\
Z & =-\frac{1}{4 n^{2}}\left[2 n(2 n-2 s-1)+s(s+1)\left(3+c^{2 n}\right)\right], \\
C_{\mathrm{V}}^{2} & =n \frac{c^{2}-1}{c^{2 n}-1} .
\end{aligned}
$$

There are in general two different values of $C_{\mathrm{S}}^{2}$ which fulfill (5.6), hence two different sound speeds. The gradient instability will be absent if

$$
C_{\mathrm{S}}^{2}>0 \text {. }
$$

In the $c \rightarrow 1$ limit Eq.(5.6) reduces to $2 \zeta\left(C_{\mathrm{S}}^{2}-1\right)^{2}=0$, in agreement with the previous result (4.21).

\footnotetext{
${ }^{9}$ Eqs. (5.5) and (5.6) are derived in the Appendix for the spatially flat $K=0$ background, but they are valid also for the spatially open $K=1$ and closed $K=-1$ backgrounds.
} 


\subsection{Simplest solutions}

Let us now consider the field equations for metrics (5.1). Eq.(2.19) yields the following non-zero components for the tensor $X_{\nu}^{\mu}$ :

$$
\begin{aligned}
X_{0}^{0} & =\frac{1}{2 n^{2}} c^{-s} \xi^{2 n-4 s}\left(n c^{2 n}-\frac{s+1}{2}\left(c^{2 n}+3\right)\right), \\
X_{1}^{1}=X_{2}^{2}=X_{3}^{3} & =\frac{1}{2 n^{2}} c^{-s} \xi^{2 n-4 s}\left(n-\frac{s+1}{2}\left(c^{2 n}+3\right)\right) .
\end{aligned}
$$

The field equations (2.22) then reduce to

$$
\begin{aligned}
\frac{3 \dot{a}^{2}}{N^{2} a^{2}}+\frac{3 K}{a^{2}} & =m^{2} \lambda_{g}+\boldsymbol{\Lambda}-m^{2} X_{0}^{0}, \\
\frac{1}{N}\left(\frac{\dot{a}}{N a}\right)^{\cdot}-\frac{K}{a^{2}} & =\frac{m^{2}}{2}\left(X_{0}^{0}-X_{1}^{1}\right) .
\end{aligned}
$$

These two equations plus an additional gauge condition do not determine all four functions $N, N_{f}, a, a_{f}$ and one of them remains free. This is the consequence of the fact that the system is undetermined because the reference metric is not yet completely specified. An extra assumption is needed in order to determine all four functions.

As the simplest option, let us assume the physical metric to be flat. There are two possibilities for this, for the flat metric of the form (5.1) can be either Minkowski,

$$
\dot{a}=0, \quad K=0,
$$

or Milne,

$$
\dot{a}=N \neq 0, \quad K=-1 .
$$

In both cases Eq.(5.11) requires that $X_{0}^{0}=X_{1}^{1}$ which implies that $c=1$. Eq.(5.10) then reduces to

$$
0=m^{2} \lambda_{g}+\Lambda-\frac{m^{2}}{2 n^{2}}(n-s-1) \xi^{2 n-4 s},
$$

which determines a constant value for $\xi$, hence the reference metric is also either Minkowski or Milne, respectively.

The Minkowski space has already been considered above. Its stability is determined by the arguments given around Eq.(4.19) - it is stable only in the FP limit corresponding to the ellipse in Fig.1.

For the Milne solution one has $\dot{a} \neq 0$ and one can apply Eq.(5.5). This gives the same result as for the de Sitter space considered above: it is stable everywhere in the region inside the ellipse in Fig.1, where the Minkowski space is unstable. This sounds odd, since the Milne space is merely a sector of Minkowski space expressed in different coordinates. If $T, R$ are the Minkowski time and radial coordinate, then one has

$$
T=a(t) \cosh (r), \quad R=a(t) \sinh (r),
$$

hence the Milne coordinates $t, r$ cover the interior of the future light cone. How can it be that the unstable Minkowski space becomes stable when expressed in different 
coordinates ? The answer is that the stability condition (5.5) guarantees the absence of ghosts in the UV limit (5.4), but there could still be soft ghosts with momenta

$$
p \leq H=\frac{\dot{a}}{N a}=\frac{1}{a}=\frac{1}{\sqrt{T^{2}-R^{2}}} .
$$

It follows that, although the Milne space does not have UV ghosts, it must contain soft ghosts with wavelengths of the order or larger than the Milne horizon. One may then argue that the Minkowski space too is actually unstable only with respect to long wave ghosts. At first glance, this contradicts the fact that the ghost modes in Minkowski space exist for any momenta. However, Eq.(4.17) shows that the leading UV contributions come from interactions of perturbations with the background curvature, which suggests that the kinetic energy of perturbations around Minkowski should be dominated by nonlinear interactions. Therefore, the linear ghost instability of Minkowski space in the UV limit could presumably be cured by the nonlinear terms.

\subsection{Solutions with constant $\xi$}

To study general solutions of Eqs.(5.10), (5.11), it is convenient to consider their consequence: the conservation condition

$$
\left(X_{0}^{0}\right)^{\cdot}=3 \frac{\dot{a}}{a}\left(X_{1}^{1}-X_{0}^{0}\right) .
$$

If $\dot{a} \neq 0$ then this replaces the second order equation (5.11). This condition can be represented in the form

$$
\frac{\dot{c}}{c}+F_{1}(c) \frac{\dot{a}}{a}+F_{2}(c) \frac{\dot{\xi}}{\xi}=0
$$

where

$$
F_{1}(c)=\frac{6 n\left(c^{2 n}-1\right)}{W}, \quad F_{2}(c)=\frac{2(n-2 s)\left((2 n-s-1) c^{2 n}-3(s+1)\right)}{W},
$$

with $W=(2 n-s-1)(2 n-s) c^{2 n}+3 s(s+1)$.

As a result, the solutions are obtained by solving Eqs.(5.10) and (5.18), supplemented by an extra condition to totally specify the system. For example, one can assume $\xi$ to be a given function of $a$, then Eq.(5.18) becomes

$$
\frac{1}{c} \frac{d c}{d a}+F_{1}(c) \frac{1}{a}+F_{2}(c) \frac{\xi^{\prime}(a)}{\xi(a)}=0
$$

integrating which yields $c=c(a)$. Injecting this to (5.9) will give $X_{0}^{0}(a)$, which will allow one to integrate Eq. (5.10).

As the simplest option, one can set $\xi(a)=\xi_{0}=$ const, in which case the spatial parts of the two metrics are proportional with the constant factor $\xi_{0}$. Eq. (5.20) then reduces to

$$
\frac{1}{c} \frac{d c}{d a}+F_{1}(c) \frac{1}{a}=0
$$


One can fulfill this by setting $c=1$, then the full 4-metrics are conformally related by the factor $\xi_{0}$. This corresponds to the solutions with proportional metrics already discussed above. The general solution of (5.21) for $c \neq$ const. is

$$
a=a_{0} \frac{c^{\alpha}}{\left(c^{2 n}-1\right)^{\beta}} \quad \text { with } \quad \alpha=\frac{s(s+1)}{2 n}, \quad \beta=\frac{n(2 n-2 s-1)+2 s(s+1)}{6 n^{2}},
$$

where $a_{0}$ is the integration constant. Inverting this to obtain $c=c(a)$ and injecting to (5.10) yields

$$
\frac{3}{N^{2}} \frac{\dot{a}^{2}}{a^{2}}+\frac{3 K}{a^{2}}=m^{2} \lambda_{g}+\Lambda-\frac{m^{2}}{4 n^{2}} \xi_{0}^{2 n-4 s}\left[(2 n-s-1) c^{2 n-s}(a)-3(s+1) c^{-s}(a)\right] .
$$

Imposing the $N=1$ gauge, this equation determines $a(t)$, which specifies all four metric amplitudes $N, N_{f}, a, a_{f}$.

\subsection{Solutions with Minkowski fiducial metric}

Let us apply the procedure outlined above to construct all solutions in the case where the reference metric is flat Minkowski. Therefore, the extra assumption is $\dot{a}_{f}=K=0$. Using the definition of $\xi$ in (5.2), one can represent (5.18) as

$$
\frac{\dot{c}}{c}+\left[F_{1}(c)-F_{2}(c)\right] \frac{\dot{a}}{a}+F_{2}(c) \frac{\dot{a}_{f}}{a_{f}}=0,
$$

and if $\dot{a}_{f}=0$ this reduces to

$$
a \frac{d c}{d a}=\frac{2 c(n-2 s-2)\left[(2 n-s) c^{2 n}-3 s\right]}{(2 n-s-1)(2 n-s) c^{2 n}+3 s(s+1)} .
$$

It is clear that

$$
c=c\left(\frac{a}{a_{0}}\right) \equiv c(\alpha)
$$

where $a_{0}$ is an integration constants, hence we set

$$
a(t)=a_{0} \alpha(t)
$$

and we also set the lapse function to the constant value,

$$
N^{2}=\frac{12 n^{2}}{m^{2}}\left(\frac{a_{f}}{a_{0}}\right)^{4 s-2 n} .
$$

Eq.(5.10) then assumes the form of the energy conservation,

$$
\frac{\dot{\alpha}^{2}}{\alpha^{2}}+\mathrm{U}(\alpha)=\mathcal{E}
$$

with the "potential energy"

$$
\mathrm{U}(\alpha)=\alpha^{4 s-2 n}\left[(2 n-s-1) c(\alpha)^{2 n-s}-3(s+1) c(\alpha)^{-s}\right],
$$


and the "total energy"

$$
\mathcal{E}=\frac{1}{3} N^{2}\left(m^{2} \lambda_{g}+\boldsymbol{\Lambda}\right)
$$

The sign of the "total energy" is determined by that of $m^{2} \lambda_{g}+\Lambda$ while its absolute value depends on $a_{0}$ and hence can be arbitrary. Solving (5.25) yields $c(\alpha)$, injecting which to (5.30) determines $\mathrm{U}(\alpha)$, and then (5.29) determines $\alpha(t)$. It is clear that $\alpha$ should be confined to the region where $\mathrm{U}(\alpha) \leq \mathcal{E}$.

The solution of the problem contains several subcases, depending on values of the parameters $n, s$. Let us first consider cases where the amplitude $c$ is constant.

I. $n=2(s+1)$. The solution of $(5.25)$ is an arbitrary constant that can be assumed to be positive, $c=c_{0}>0$. The potential becomes

$$
\mathrm{U}=\frac{3(s+1) c_{0}^{-s}\left(c_{0}^{4(s+1)}-1\right)}{\alpha^{4}}
$$

which can be positive or negative, depending on values of $s$ and $c_{0}$. If $\mathrm{U}$ is negative, then it describes an effective radiation mimicked by massive gravitons. The scale factor then evolves as in the universe containing a radiation and an effective cosmological term mimicked by $\mathcal{E}$. If $\mathrm{U}>0$ then the massive gravitons mimic a "phantom radiation". One has in this case $\mathrm{U}(\alpha) \rightarrow+\infty$ as $\alpha \rightarrow 0$ hence there is an infinite potential barrier near singularity so that the solution is a bounce: the universe first shrinks up to a minimal non-zero size, then hits the potential barrier and expands again. It is worth noting that the very existence of bounces indicates that the Null Energy Condition is violated.

At the same time, the solutions can be free of ghosts and gradient instabilities. Indeed, choosing $c_{0}$ to be close to unity, the no-ghost condition $\zeta\left(c_{0}\right)>0$ expressed by (5.5) will have approximately the same solutions as for $c_{0}=1$, corresponding to the interior of the ellipse in Fig.1. The two sound speeds will then be close to unity.

II. $n \neq 2(s+1), c=$ const. The solution of (5.25) in this case is

$$
c^{2 n}=\frac{3 s}{2 n-s} \equiv c_{*}^{2 n},
$$

where one should assume that either $2 n>s>0$ or $2 n<s<0$ for $c_{*}$ to be positive. One has

$$
\mathrm{U}=-\frac{6 n c_{*}^{-s}}{2 n-s} \alpha^{4 s-2 n}<0 .
$$

If $n=2 s$ then $\mathrm{U}=-4$ and the universe expands with a constant Hubble expansion rate. If $n>2 s$ then for small $\alpha$ the universe is dominated by an effective "fluid" mimicked by $\mathrm{U}$ while for large $\alpha$ the potential approaches zero and the universe expands with a constant Hubble expansion rate $H^{2}=\mathcal{E} / N^{2}$ (which should be positive). If $n<2 s$ then the "fluid term" U grows without bound as $\alpha \rightarrow \infty$.

The no-ghost condition (5.4) reduces to

$$
\zeta\left(c_{*}\right)=-\frac{3 s}{2 n}>0,
$$


which is impossible to fulfill since $n$ and $s$ should be either both positive or both negative for $c_{*}$ to be positive. Therefore, type II solutions always have ghost.

III. $n \neq 2(s+1), c \neq$ const. Let us now study solutions for which the amplitude $c$ is not constant. The general solution of $(5.25)$ for $n \neq 2(s+1)$ is

$$
\frac{(2 n-s) c^{2 n}-3 s}{c^{s+1}}=\left(\frac{a}{a_{0}}\right)^{2(n-2 s-2)} .
$$

Several subcases are to be considered. The left hand side of this expression is positive in one of the following cases,
(a) $s=2 n<0$;
(b) $s=0, n>0$;
(c) $n>s / 2>0, c^{2 n}>3 s /(2 n-s)$;
(d) $n<s / 2<0, c^{2 n}<|3 s /(2 n-s)|$;
(e) $n>s / 2, s<0$.

Each of these cases further splits into subcases depending on the signs of $n$, of $s+1$, of $2 n-s-1$, and of $n-2 s-2$. This renders the classification of solutions a bit tedious, but still manageable.

IIIa. Let us first consider case (a) in (5.37), where $s=2 n<0$. Then, properly redefining the constant $a_{0}$ in (5.36), one obtains

$$
c^{2 n+1}=\left(\frac{a}{a_{0}}\right)^{2(3 n+2)} \equiv \alpha^{2(3 n+2)},
$$

hence, as $\alpha$ increases from zero to infinity, $c$ either increases from zero to infinity or decreases from infinity to zero. The potential is

$$
\mathrm{U}(\alpha)=-\alpha^{6 n}-3(2 n+1) \alpha^{-\frac{2 n}{2 n+1}} .
$$

Depending on value of $n$ there are several subcases.

IIIa-1: $-1 / 2<n<0, s=2 n$. $c(\alpha)$ increases as $\alpha$ increases. The potential $\mathrm{U}(\alpha)$ is always negative and its absolute value becomes large for $\alpha \rightarrow 0$ and for $\alpha \rightarrow \infty$.

IIIa-2: $-2 / 3<n<-1 / 2, s=2 n$. $c(\alpha)$ decreases as $\alpha$ increases. The potential $\mathrm{U}(\alpha)$ is qualitatively similar to the one shown in panel B in Fig. 2 below. It is large and positive at small $\alpha$ while for $\alpha \rightarrow \infty$ it approaches zero from below, hence there is a minimal value $\mathrm{U}_{\min }=\mathrm{U}\left(\alpha_{\min }\right)<0$ with $\alpha_{\text {min }}=1$. If $\mathcal{E}=\mathrm{U}_{\text {min }}$ then the system always rests at the minimum and the geometry is flat with $c\left(\alpha_{\min }\right)=1$. If $\mathrm{U}_{\text {min }}<\mathcal{E}<0$ then $\alpha(t)$ oscillates around the value $\alpha_{\min }$ while $c(t)$ oscillates around $c\left(\alpha_{\min }\right)=1$. For $\mathcal{E} \geq 0$ the solution is a bounce.

IIIa-3: $n<-2 / 3, s=2 n . \quad c(\alpha)$ increases as $\alpha$ increases. The potential $\mathrm{U}(\alpha)$ is qualitatively similar to the one shown in panel $\mathrm{A}$ in Fig.2 below. It is large and negative for small $\alpha$, then passes through a maximal value $\mathrm{U}_{\max }=\mathrm{U}\left(\alpha_{\max }\right)>0$ with $\alpha_{\max }=1$, then approaches zero from above as $\alpha \rightarrow \infty$. Depending on value of $\mathcal{E}$, the motions in this potential correspond either to cosmologies with an initial singularity or to non-singular bounces. If $\mathrm{U}_{\max }=\mathcal{E}$ then there is a solution for which $\alpha(t)$ grows from the constant value $\alpha_{\max }$ in the past to infinity in the future, hence the universe interpolates between the flat space and de Sitter space. 
There are also two boundary cases, $n=-1 / 2$ and $n=-2 / 3$. For $n=-1 / 2$ the scale factor $a$ should be constant, as seen from Eq.(5.25), hence this is a particular case of the Minkowski solutions. The $n=-2 / 3$ case corresponds to the intersection with family I since one has then $n=2(s+1)$.

For all type IIIa solutions the no-ghost condition (5.5) reduces to

$$
\zeta(c)=-3 \frac{2 n+1}{2 n}>0,
$$

which is independent of $c$. This condition is fulfilled for solutions of type IIIa-1, hence they are ghost-free. However, one finds then that the sound speeds are imaginary, hence there are gradient instabilities. The no-ghost condition is violated for solutions of types IIIa-2 and IIIa-3.

IIIb. In case (b) in (5.37), for $s=0$ and $n>0$, the no-ghost condition (5.5) becomes

$$
\zeta(c)=\frac{1-2 n}{2 n} c^{2 n}>0 .
$$

This is satisfied for $n<1 / 2$, however, there are gradient instabilities in this case as the coefficient of $C_{S}^{2}$ in (5.6) is positive.

IIIc. Let us now consider case (c) in (5.37), with $n>s / 2>0$. Then, redefining the integration constant $a_{0}$, Eq.(5.36) can be rewritten as

$$
\frac{c^{2 n}-c_{*}^{2 n}}{c^{s+1}}=\left(\frac{a}{a_{0}}\right)^{2(n-2 s-2)} \equiv \alpha^{2(n-2 s-2)},
$$

where $c_{*}$ is the same as in (5.33), hence one should have $c \in\left[c_{*}, \infty\right)$ for the left hand side to be positive. There are again several cases to study and one finds the following possibilities.

IIIc-1: $2 n>s>0, n>2(s+1) . c(\alpha)$ increases from $c_{*}$ to infinity; the potential $\mathrm{U}(\alpha)$ has a maximum as shown in panel A in Fig.2.

IIIc-2: $2 n>s>0, \max \{(s+1) / 2,2 s\}<n<2(s+1)$. $c(\alpha)$ decreases from infinity to $c_{*}$; the potential $\mathrm{U}(\alpha)$ has a minimum as shown in panel B in Fig.2.

IIIc-3: $2 n>s>0,(s+1) / 2<n<2 s$. Both $c(\alpha)$ and $\mathrm{U}(\alpha)$ decrease as shown in panel C in Fig.2.

IIIc-4: $2 n>s>0, s / 2<n<(s+1) / 2$. Both $c(\alpha)$ and $\mathrm{U}(\alpha)$ are two-valued functions defined only for $\alpha \geq \alpha_{\min }>0$ as shown in panel D in Fig.2. For $\mathcal{E}<\mathrm{U}_{\max }$ there are two different bounce solutions corresponding to reflections from either the lower or upper branch of $U$.

For solutions of types IIIc-1, IIIc-2, and IIIc-3 the amplitude $c(\alpha)$ approaches the value $c_{*}$ either for small or for large $\alpha$, which insures that there is ghost since $\zeta\left(c_{*}\right)=-(3 s) /(2 n)<0$ in these cases.

The situation is more complex in case IIIc-4. If one plots $a, \mathrm{U}$, and $\zeta(c)$ against $c$ in this case, one finds that $a(c)$ attains a minimal non-zero value at some $c_{m}$, the potential $\mathrm{U}(c)$ attains a maximum at the same time, while $\zeta(c)$ changes sign. As a result, both $c(\alpha)$ and $\mathrm{U}(\alpha)$ are double-valued functions as shown in panel D in Fig.2. Their two branches determine two different solutions which should be considered independently. 

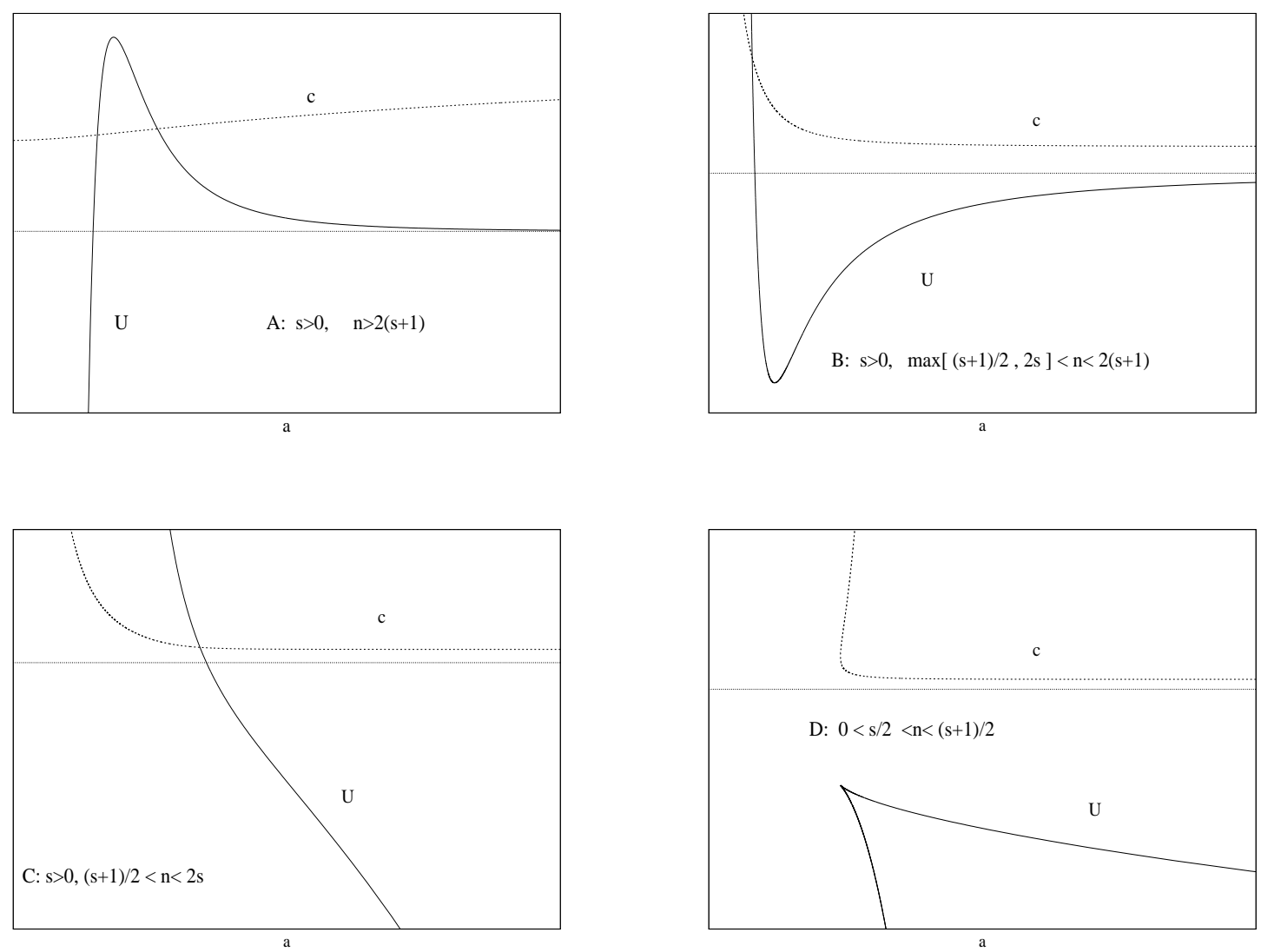

Figure 2. The potential $\mathrm{U}(\alpha)$ and $c(\alpha)$ for generic $2 n>s>0$.

The upper branch of $\mathrm{U}(\alpha)$ corresponds to the lower branch of $c(\alpha)$ where one has $\zeta(c)<0$, hence there is ghost. The lower branch of $\mathrm{U}(\alpha)$ corresponds to the upper branch of $c(\alpha)$ where $\zeta(c)>0$, hence this branch is ghost-free. However, one finds gradient instabilities there.

IIId and IIIe. Nothing qualitatively new is found in cases (d) and (e) in (5.37). There are again several subcases to study, but each time one finds the potential to be either of one of the types show in Fig.2, or of type IIIa-1. All of these solutions contain ghosts and/or gradient instabilities.

This gives all solutions with the Minkowski reference metric. Only type I solutions can be stable.

\subsection{Solutions with Milne fiducial metric}

If the fiducial metric is Milne, then $K=-1, N_{f}=\dot{a}_{f}$, and Eq.(5.24) reduces to

$$
\frac{\dot{c}}{c}+\left[F_{1}(c)-F_{2}(c)\right] \frac{\dot{a}}{a}+F_{2}(c) c \frac{N}{a}=0 .
$$


Setting $c$ to a constant value yields

$$
\frac{\dot{a}}{N}=\frac{c F_{2}(c)}{F_{2}(c)-F_{1}(c)},
$$

injecting which to (5.10) with $K=-1$ one obtains

$3\left(\left[\frac{c F_{2}(c)}{F_{2}(c)-F_{1}(c)}\right]^{2}-1\right) \frac{1}{a^{2}}=m^{2} \lambda_{g}+\Lambda-\frac{m^{2}}{4 n^{2}} c^{-s}\left[(2 n-s-1) c^{2 n}-3(s+1)\right] \xi^{2 n-4 s}$.

This equation determines $\xi=\xi(a)$, while (5.44), imposing the gauge where $N=1$, insures that $a$ is a linear function of time. This specifies all functions in the problem. If $c \neq 1$ then the physical metric is not flat. If $c$ is close to unity then one can adjust $s, n$ such that the solution will be free of ghosts and tachyons (in the UV limit).

More general solutions with $\dot{c} \neq 0$ can be obtained by setting $N=a$ in (5.43), which gives the equation containing only $c$ and $a$. However, solutions of this equations are not immediately obvious. At the same time, as we learned above, solutions with a non-trivial $c$ usually show ghosts and/or gradient instabilities.

At this point, we terminate our analysis of the OP theory and come to the conclusions.

\section{Summary and concluding remarks}

We presented above our analysis of the theory constructed by Ogievetsky and Polubarinov in [1]. Taking apart the remarkable way it was obtained, it is just a bimetric massive gravity with a specially designed mass term. As any other bimetric theory, it has two tensors, $X_{\nu}^{\mu}$ and $Y_{\nu}^{\mu}$, obtained by varying with respect to the two metrics, respectively. If the reference metric is flat, then one has on-shell $\partial_{\mu} Y_{\nu}^{\mu}=0$. The specialty of the OP theory is that, by the non-linear field redefinition (1.11), its physical metric can be algebraically expressed in terms of the "graviton field" $\Psi^{\mu \nu}$ in such a way that $Y_{\nu}^{\mu}$ becomes linear in $\Psi^{\mu \nu}$. Therefore, the field equations imply as a consequence the linear "spin limitation condition" $\partial_{\mu} \Psi^{\mu}{ }_{\nu}+q \partial_{\nu} \Psi_{\alpha}^{\alpha}=0$. In the OP's view, this property is very important as it allows one to carry out a "clean" classification of the spin states present in the theory. The linear subsidiary condition arising on-shell is the key property that distinguishes the OP theory among other bimetric models.

It is, however, not immediately obvious what this property gives in practical terms. The most striking point is that the OP theory has a non-zero intersection with the dRGT ghost-free massive gravity. However, for generic parameter values it propagates 6 degrees of freedom and shows ghost on flat background. According to the currently adopted view, these properties are unacceptable. Nevertheless, we studied the phenomenology of the theory to see if something interesting may emerge.

We found many different types of homogeneous and isotropic cosmological solutions, including self-accelerating cosmologies, bounces, oscillating solutions, etc. Unfortunately, most of them show ghost and/or gradient instabilities. Surprizingly, however, 
we find that the de Sitter space is stable in a large region of the parameter space, in spite of the presence of the 6-th degree of freedom, hence the Boulware-Deser mode becomes benign. Moreover, even the instability of the flat space does not seem so dramatic, as it turns out that the Milne universe, which is a sector of Minkowski space, is actually UV stable in the theory. Therefore, the instability can only be due to the soft modes with wavelengths of the order or larger than the Milne horizon. This suggests that the flat space ghost instability is similar to the classical Jeans instability with respect to long-wave perturbations. Therefore, the BD mode could probably be viewed as some kind of non-relativistic fluid [26].

Our conclusion is that the OP theory does show interesting features, despite the presence of the 6-th polarization. This may be due to the "spin limitation" encoded in the theory, even though there are other massive gravities with a healthy 6 -th mode [27]. It would be interesting to study also other solutions in the theory, as for example black holes. It seems also that the particular OP theory with $n=1 / 2$ and $s=0$, which shows both the "spin limitation" and the "freedom of the ghost", should be further studied.

\section{Acknowledgements}

The work of S.M. was supported by Japan Society for the Promotion of Science (JSPS) Grants-in-Aid for Scientific Research (KAKENHI) No. 17H02890, No. 17H06359, and by World Premier International Research Center Initiative (WPI), MEXT, Japan. M.S.V. thanks for hospitality the YITP in Kyoto, where a part of this work was completed. Discussions during the workshop YITP-T-17-02 "Gravity and Cosmology 2018" and the YKIS2018a symposium "General Relativity - The Next Generation" were useful. His work was also partly supported by the Russian Government Program of Competitive Growth of the Kazan Federal University.

\section{A Stability of more general cosmologies}

In this Appendix we derive the stability conditions for cosmological solutions described by metrics (5.1). These metrics determine the matrices

$$
S_{\nu}^{\mu}=\xi^{2}\left(\begin{array}{cc}
y^{2} & 0 \\
0 & \mathbb{1}_{3}
\end{array}\right), \quad \Sigma_{\nu}^{\mu}=\left(\hat{S}^{n}\right)^{\mu}{ }_{\nu}=\xi^{2 n}\left(\begin{array}{cc}
y^{2 n} & 0 \\
0 & \mathbb{1}_{3}
\end{array}\right) .
$$

Perturbing the metrics via

$$
g_{\mu \nu} \rightarrow g_{\mu \nu}+h_{\mu \nu}, \quad f_{\mu \nu} \rightarrow f_{\mu \nu},
$$

one has (with $i, j=1,2,3$ )

$$
\delta S^{\mu}{ }_{\nu}=-g^{\mu \alpha} h_{\alpha \sigma} S^{\sigma}{ }_{\nu} \equiv\left(\begin{array}{cc}
\delta S_{0}^{0} & \delta S^{0}{ }_{j} \\
\delta S^{i}{ }_{0} & \delta S^{i}{ }_{j}
\end{array}\right) .
$$


This does not commute with $S_{\nu}^{\mu}$, which complicates the computation of $\delta \Sigma^{\mu}{ }_{\nu}=\delta\left(\hat{S}^{n}\right)^{\mu}{ }_{\nu}$. However, a direct calculation yields

$$
\hat{S}^{k} \delta \hat{S} \hat{S}^{m}=\xi^{2 k+2 m}\left(\begin{array}{ccc}
y^{2 m+2 k} & \delta S^{0}{ }_{0} & y^{2 k} \delta S^{0}{ }_{j} \\
y^{2 m} \delta S^{i}{ }_{0} & \delta S^{i}{ }_{j}
\end{array}\right)
$$

Assuming for a moment $n$ to be integer, one has

$$
\delta \hat{\Sigma}=\delta\left(\hat{S}^{n}\right)=\delta \hat{S} \hat{S}^{n-1}+\hat{S} \delta \hat{S} \hat{S}^{n-2}+\ldots+\hat{S}^{n-1} \delta \hat{S}
$$

and using (A.4) one can sum up the geometric series, which yields

$$
\delta \Sigma_{\nu}^{\mu}=\xi^{2 n-2}\left(\begin{array}{ccc}
n y^{2 n-2} \delta S^{0}{ }_{0} & \gamma \delta S^{0}{ }_{j} \\
\gamma \delta S^{i}{ }_{0} & n & \delta S^{i}{ }_{j}
\end{array}\right) \quad \text { with } \quad \gamma=\frac{y^{2 n}-1}{y^{2}-1} .
$$

We now simply extend this expression to arbitrary real values of $n$ and inject it to

$$
\delta X^{\mu}{ }_{\nu}=\frac{s}{2} h X_{\nu}^{\mu}+\frac{1}{2 n^{2}} \phi^{s}\left(n \delta \Sigma^{\mu}{ }_{\nu}-\frac{s+1}{2} \delta \Sigma^{\alpha}{ }_{\alpha} \delta^{\mu}{ }_{\nu}\right)
$$

to compute

$$
\delta X_{\mu \nu}=h_{\mu \alpha} X_{\nu}^{\alpha}+g_{\mu \alpha} X_{\nu}^{\alpha}
$$

which is to be used in the perturbation equations (3.7). We use the representation (4.4) of $h_{\mu \nu}$ in terms of the scalar, vector, and tensor modes to insert to (A.3) to obtain $\delta S^{\mu}$, which is then used in (A.4) and in (A.7)-(A.8) to obtain $E_{\mu \nu}$, and finally in (4.9) to obtain the effective action.

The effective action splits again into the sum of the tensor, vector, and scalar parts. In the tensor sector we obtain

$$
I_{\mathrm{T}}=\frac{M_{\mathrm{Pl}}^{2}}{4} \int\left(\frac{1}{N^{2}}\left(\dot{D}_{1}^{2}+\dot{D}_{2}^{2}\right)-\left[M_{\mathrm{T}}^{2}+\frac{p^{2}}{a^{2}}\right]\left(D_{1}^{2}+D_{2}^{2}\right)\right) N a^{3} d t d^{3} x
$$

which is completely similar to the expression (4.10) we had before, the only difference being the change in the mass parameter value,

$$
M^{2}=m^{2} \xi^{2 n-4 s} \rightarrow M_{\mathrm{T}}^{2}=m^{2} \xi^{2 n-4 s} c^{-s},
$$

which becomes a function of time since $\xi$ and $c$ are now time-dependent.

In the vector sector we obtain

$$
I_{\mathrm{V}}=\frac{M_{\mathrm{Pl}}^{2}}{4 \mu^{2}} \int_{\mathrm{T}}\left(\frac{p^{2}}{N^{2}\left[C_{\mathrm{V}}^{2} p^{2} / a^{2}+M_{\mathrm{T}}^{2}\right]}\left(\dot{V}_{1}^{2}+\dot{V}_{2}^{2}\right)-p^{2}\left(V_{1}^{2}+V_{2}^{2}\right)\right) N a^{3} d t d^{3} x
$$

with

$$
C_{\mathrm{V}}^{2}=n \frac{c^{2}-1}{c^{2 n}-1}
$$

This action reduces to (4.11) when $c \rightarrow 1$. 
In the scalar sector we find

$$
I_{\mathrm{S}}=\frac{M_{\mathrm{Pl}}^{2}}{4} \int M_{\mathrm{T}}^{2} \sum_{a, b=1,2}\left(\frac{1}{N^{2}} K_{a b} \dot{S}_{a} \dot{S}_{b}+\frac{\mathcal{Q}}{N} \epsilon_{a b} \dot{S}_{a} S_{b}-U_{a b} S_{a} S_{b}\right) N a^{3} d t d^{3} x
$$

where the expressions for $K_{a b}, \mathcal{Q}$, and $U_{a b}$ are rather complicated, but they simplify for $p \rightarrow \infty$. We obtain

$$
K_{11}=\frac{\zeta(c)}{2 \mathcal{H}^{2}}+\mathcal{O}\left(1 / p^{2}\right), \quad K_{22}=\frac{a^{2} p^{2}}{4 \mu^{4} C_{\mathrm{V}}^{2}}+\mathcal{O}(1), \quad K_{12}=-\frac{\zeta(c)}{C_{\mathrm{V}}^{2}} \frac{M_{\mathrm{T}}^{2} a^{2}}{8 \mu^{2} \mathcal{H}^{2}}+\mathcal{O}\left(1 / p^{2}\right)
$$

where $\mathcal{H} \equiv \dot{a} /(N a)$ is the Hubble parameter of the background metric and

$$
\zeta(c) \equiv-\frac{(2 n-s-1)(2 n-s) c^{2 n}+3 s(s+1)}{4 n^{2}} .
$$

One has

$$
\operatorname{tr}\left(K_{a b}\right)=\frac{a^{2} p^{2}}{4 \mu^{4} C_{\mathrm{V}}^{2}}+\mathcal{O}(1), \quad \operatorname{det}\left(K_{a b}\right)=\frac{\zeta(c) a^{2} p^{2}}{8 \mu^{4} C_{\mathrm{V}}^{2} \mathcal{H}^{2}}+\mathcal{O}(1)
$$

and the positivity of these expressions, which guarantees the absence of ghost, yields a single condition,

$$
\zeta(c)>0
$$

This explains Eq.(5.4) in the main text.

One can also compute the sound speed using (4.20). The necessary elements are

$$
\mathcal{Q}=-\frac{\omega}{4 \mu^{2} \mathcal{H}} p^{2}+\mathcal{O}(1)
$$

where

$$
\omega=-\frac{1+c^{2}}{2 C_{\mathrm{V}}^{2}}+\frac{2 s+1}{2 n}\left(1+c^{2 n}\right)-\frac{s(s+1)}{2 n^{2}}\left(3+c^{2 n}\right) \stackrel{c \rightarrow 1}{\longrightarrow} 1+2 \zeta .
$$

One also uses

$$
U_{11}=-\frac{c^{2} p^{2}}{4 a^{2} C_{\mathrm{V}}^{2} \mathcal{H}^{2}}+\mathcal{O}(1), \quad U_{22}=-\frac{Z}{2 \mu^{4}} p^{4}+\mathcal{O}\left(p^{2}\right), \quad U_{12}=\mathcal{O}\left(p^{2}\right),
$$

where

$$
Z=-\frac{1}{4 n^{2}}\left[2 n(2 n-2 s-1)+s(s+1)\left(3+c^{2 n}\right)\right] \stackrel{c \rightarrow 1}{\longrightarrow} \zeta .
$$

This is enough to compute the sound speed in the UV limit. Eq.(4.20) yields in the leading in $p$ order the equation for $C_{\mathrm{S}}^{2}$,

$$
2 C_{\mathrm{V}}^{2} \zeta(c) C_{\mathrm{S}}^{4}+\left[C_{\mathrm{V}}^{4}\left(4 Z \zeta(c)-\omega^{2}\right)+c^{2}\right] C_{\mathrm{S}}^{2}+2 c^{2} C_{\mathrm{V}}^{2} Z=0,
$$


which gives rise to Eq.(5.6) in the main text.

We obtained the above conditions assuming that the spatial curvature in (5.1) vanishes, $K=0$. However, since they are derived in the UV limit, they must be sensitive only to the local physics, which is the same for all values of $K$, hence they are expected to be valid for any $K$. Rigorously speaking, it is possible that the dimensionless combination $\left(m^{2} \lambda_{g}+\boldsymbol{\Lambda}-m^{2} X_{0}^{0}\right) N^{2} a^{2} / \dot{a}^{2}-3\left(=3 N^{2} K / \dot{a}^{2}\right)$, which vanishes for $K=0$, might appear in the no-ghost condition and the sound speed. Nonetheless, explicit calculations with $K \neq 0$ show that this is not the case. As a result, the no-ghost condition (5.5) and the algebraic equation (5.6) that determines the sound speed are valid for any $K$.

\section{References}

[1] V. I. Ogievetsky and I. V. Polubarinov, Interacting field of spin 2 and the Einstein equations, Ann.Phys. 35 (1965) 167-208.

[2] M. Fierz and W. Pauli, On relativistic wave equations for particles of arbitrary spin in an electromagnetic field, Proc.Roy.Soc.Lond. A173 (1939) 211-232.

[3] C. de Rham, G. Gabadadze and A. Tolley, Resummation of massive gravity, Phys.Rev.Lett. 106 (2011) 231101, [1011.1232].

[4] A. Papapetrou, Einstein's theory of gravitation and flat space, Proc. Roy. Irish Acad.(Sect. A) 52A (1948) 11-23.

[5] S. Gupta, Einstein's and other theories of gravitation, Rev.Mod.Phys. 29 (1957) 334-336.

[6] R. P. Feynman, Quantum theory of gravitation, Acta Phys. Polon. 24 (1963) 697-722.

[7] E. Poisson and C. M. Will, Gravity: Newtonian, Post-Newtonian, Relativistic. Cambridge University Press, 2014.

[8] S. Deser, Selfinteraction and gauge invariance, Gen. Rel. Grav. 1 (1970) 9-18, [gr-qc/0411023].

[9] D. G. Boulware and S. Deser, Can gravitation have a finite range?, Phys.Rev. D6 (1972) 3368-3382.

[10] C. Mazuet and M. S. Volkov, Massive spin-2 field in arbitrary spacetimes the detailed derivation, JCAP 1807 (2018) 012, [1804.01970].

[11] A. De Felice, E. A. Gumrukcuoglu and S. Mukohyama, Massive gravity: nonlinear instability of the homogeneous and isotropic universe, Phys.Rev.Lett. 109 (2012) 171101, [1206.2080].

[12] M. Fasiello and A. J. Tolley, Cosmological stability bound in massive gravity and bigravity, JCAP 1312 (2013) 002, [1308.1647].

[13] A. H. Chamseddine and V. Mukhanov, Hidden ghost in massive gravity, JHEP 1303 (2013) 092, [1302.4367].

[14] H. van Dam and M. J. G. Veltman, Massive and massless Yang-Mills and gravitational fields, Nucl. Phys. B22 (1970) 397-411. 
[15] A. I. Vainshtein, To the problem of nonvanishing gravitation mass, Phys.Lett. B39 (1972) 393-394.

[16] Ya. B. Zeldovich and L. P. Grishchuk, Gravitation, the General Theory of Relativity, and alternative theories, Sov. Phys. Usp. 29 (1986) 780-787.

[17] A. Maheshwari, Spin-2 field theories and the tensor-field identity, Nuovo Cim. A8 (1972) 319-330.

[18] J. B. Pitts, Universally coupled massive gravity, III: dRGT-Maheshwari pure spin-2, Ogievetsky-Polubarinov and arbitrary mass terms, Annals Phys. 365 (2016) 73-90, [1505.03492].

[19] E. A. Ivanov and J. Niederle, Gauge formulation of gravitation theories. 1. The Poincare, de Sitter and conformal cases, Phys. Rev. D25 (1982) 976.

[20] C. Cutler and R. M. Wald, A new type of gauge invariance for a collection of massless spin-2 fields. 1. Existence and uniqueness, Class. Quant. Grav. 4 (1987) 1267.

[21] E. T. Tomboulis, Exact relation between Einstein and quadratic quantum gravity, Phys. Lett. B389 (1996) 225-230, [hep-th/9601082].

[22] N. Boulanger, T. Damour, L. Gualtieri and M. Henneaux, Inconsistency of interacting, multigraviton theories, Nucl. Phys. B597 (2001) 127-171, [hep-th/0007220].

[23] Yu. M. Zinoviev, On massive spin 2 interactions, Nucl. Phys. B770 (2007) 83-106, [hep-th/0609170].

[24] T. R. Morris, Renormalization group properties of the conformal sector: towards perturbatively renormalizable quantum gravity, 1802.04281.

[25] A. Higuchi, Forbidden mass range for spin-2 field theory in de Sitter space-time, Nucl.Phys. B282 (1987) 397.

[26] A. E. Gumrukcuoglu, S. Mukohyama and T. P. Sotiriou, Low energy ghosts and the Jeans instability, Phys. Rev. D94 (2016) 064001, [1606.00618].

[27] M. Celoria, D. Comelli and L. Pilo, Fluids, superfluids and supersolids: dynamics and cosmology of self gravitating media, JCAP 1709 (2017) 036, [1704.00322]. 